\title{
Elastic contact mechanics: Percolation of the contact area and fluid squeeze-out
}

\author{
B.N.J. Persson ${ }^{1, a}$, N. Prodanov ${ }^{1,2}$, B.A. Krick ${ }^{3}$, N. Rodriguez ${ }^{4}$, N. Mulakaluri ${ }^{1}$, W.G. Sawyer ${ }^{3}$, and P. Mangiagalli ${ }^{5}$ \\ 1 IFF, FZ Jülich, D-52425 Jülich, Germany \\ 2 Sumy State University, 2 Rimskii-Korsakov Str., 40007 Sumy, Ukraine \\ 3 Department of Mechanical and Aerospace Engineering, University of Florida, Gainesville FL 32611, USA \\ 4 Advanced Technologies, BD Medical-Pharmaceutical Systems, 1 Becton Drive, MC 427, Franklin Lakes, NJ 07417, USA \\ ${ }^{5}$ Advanced Technologies, BD-Pharmaceutical Systems, 38800 Pont de Claix, France
}

Received 7 November 2011 and Received in final form 5 January 2012

Published online: 26 January 2012 - (C) EDP Sciences / Società Italiana di Fisica / Springer-Verlag 2012

\begin{abstract}
The dynamics of fluid flow at the interface between elastic solids with rough surfaces depends sensitively on the area of real contact, in particular close to the percolation threshold, where an irregular network of narrow flow channels prevails. In this paper, numerical simulation and experimental results for the contact between elastic solids with isotropic and anisotropic surface roughness are compared with the predictions of a theory based on the Persson contact mechanics theory and the Bruggeman effective medium theory. The theory predictions are in good agreement with the experimental and numerical simulation results and the (small) deviation can be understood as a finite-size effect. The fluid squeeze-out at the interface between elastic solids with randomly rough surfaces is studied. We present results for such high contact pressures that the area of real contact percolates, giving rise to sealed-off domains with pressurized fluid at the interface. The theoretical predictions are compared to experimental data for a simple model system (a rubber block squeezed against a flat glass plate), and for prefilled syringes, where the rubber plunger stopper is lubricated by a high-viscosity silicon oil to ensure functionality of the delivery device. For the latter system we compare the breakloose (or static) friction, as a function of the time of stationary contact, to the theory prediction.
\end{abstract}

\section{Introduction}

The influence of surface roughness on fluid flow at the interface between solids in stationary or sliding contact is a topic of great importance both in nature and technology. Technological applications include leakage of seals, mixed lubrication, and removal of water from the tire-road footprint. In nature, fluid removal (squeeze-out) is important for adhesion and grip between the tree frog or gecko adhesive toe pads and the countersurface during raining, and for cell adhesion.

Almost all surfaces in nature and most surfaces of interest in tribology have roughness on many different length scales, sometimes extending from atomic distances $(\sim 1 \mathrm{~nm})$ to the macroscopic size of the system which could be of order $\sim 1 \mathrm{~cm}$. Often the roughness is fractallike so that when a small region is magnified (in general with different magnification in the parallel and orthogonal directions) it "looks the same" as the unmagnified surface.

Most objects produced in engineering have some particular macroscopic shape characterized by a radius of

\footnotetext{
a e-mail: b.persson@fz-juelich.de
}

curvature (which may vary over the surface of the solid) e.g., the radius $R$ of a cylinder in a combustion engine. In this case the surface may appear perfectly smooth to the naked eye, but at short enough length scale, in general much smaller than $R$, the surface will exhibit strong irregularities (surface roughness). The surface roughness power spectrum $C(\mathbf{q})$ of such a surface will exhibit a rolloff wavelength $\lambda_{0} \ll R$ (related to the roll-off wave vector $q_{0}=2 \pi / \lambda_{0}$ ) and will appear smooth (except for the macroscopic curvature $R$ ) on length scales much longer than $\lambda_{0}$. In this case, when studying the fluid flow between two macroscopic solids, one may homogenize the microscopic fluid dynamics occurring at the interface, resulting in effective fluid flow equations describing the average fluid flow on length scales much larger than $\lambda_{0}$, and which can be used to study, e.g., the lubrication of the cylinder in an engine. This approach of eliminating or integrating out short length scale degrees of freedom to obtain effective equations of motion which describe the long distance (or slow) behavior is a very general and powerful concept often used in physics, and is employed in the study presented below. 
In the context of fluid flow at the interface between closely spaced solids with surface roughness, Patir and Cheng $[1,2]$ have shown how the Navier-Stokes equations of fluid dynamics can be reduced to effective equations of motion involving locally averaged fluid pressure and flow velocities. In the effective equation the so-called flow factors occur, which are functions of the locally averaged interfacial separation $\bar{u}$. The authors showed how the flow factors can be determined by solving numerically the fluid flow in small rectangular units with linear size of order of (or larger than) the roll-off wavelength $\lambda_{0}$ introduced above, and by averaging over several realizations. However, with the present speed (and memory) limitations of computers fully converged solutions using this approach can only take into account roughness over two or at most three decades in length scale. In addition, Patir and Cheng did not include the long-range elastic deformations of the solid walls in the analysis. Later studies have attempted to include elastic deformation using asperity contact mechanics models as pioneered by Greenwood-Williamson (GW) [3], but it is now known that this theory (and other asperity contact models [4]) does not accurately describe contact mechanics because of the neglect of the long-range elastic coupling between the asperity contact regions $[5,6]$. In particular, the relation between the average interfacial separation $\bar{u}$ and the squeezing pressure $p$, which is very important for the fluid flow problem, is not accurately described by the GW model [7-9].

The paper by Patir and Cheng was followed by many other studies of how to eliminate or integrate out the surface roughness in fluid flow problems (see, e.g., the work by Sahlin et al. [10]). Most of these theories involve solving numerically the fluid flow in rectangular interfacial units and, just as in the Patir and Cheng approach, cannot include roughness on more than $\sim 2$ decades in length scale. In addition, in some of the studies the measured roughness topography must be "processed" in a non-trivial way in order to obey periodic boundary conditions (which is necessary for the Fast Fourier Transform method used in some of these studies).

Tripp [11] has presented an analytical derivation of the flow factors for the case where the separation between the surfaces is so large that no direct solid-solid contact occurs. He obtained the flow factors to first order in $\left\langle h^{2}\right\rangle / \bar{u}^{2}$, where $\left\langle h^{2}\right\rangle$ is the ensemble average of the square of the roughness amplitude and $\bar{u}$ is the average surface separation. The result of Tripp has recently been generalized to include elastic deformations of the solids $[12,13]$.

In this paper, the study of fluid squeeze-out from the region between two elastic solids with randomly rough surfaces is presented. We focus on such high contact pressures that after long enough contact time the area of real contact percolates resulting in pockets of confined, pressurized, fluid at the interface. The Bruggeman effective medium theory and the Persson contact mechanics theory are employed to calculate the interfacial fluid conductivity tensor. For anisotropic surface roughness the Bruggeman effective medium theory predicts that the contact area percolates when $A / A_{0}=\gamma /(1+\gamma)$, where $\gamma$ is the Peklenik number (the ratio between the decay length of the heightheight correlation function along the two principle directions) and $A / A_{0}$ is the relative contact area ( $A_{0}$ is the nominal or apparent contact area). The main aim of the present work is to verify the theory predictions through the comparison with the results of molecular dynamics (MD) simulations and experiments. MD calculations have been carried out both for isotropic and anisotropic surface roughness, while the experiments consider only the surfaces with isotropic statistical properties (where $\gamma=1$ ). The paper outline is as follows. The theoretical approach and its application to the fluid squeeze-out are described in sects. 2-4 and 5, respectively. Sections 6 and 7 present simulations and experiments. In sect. 8 we apply the theory to the breakloose (or static) friction for prefillable syringes. The work is closed by the concluding sect. 9 .

\section{Anisotropic surface roughness}

Many surfaces of practical importance have roughness with isotropic statistical properties, e.g., sandblasted surfaces or surfaces coated with particles typically bound by a resin to an otherwise flat surface, e.g., sandpaper surfaces. However, some surfaces of engineering interest have surface roughness with anisotropic statistical properties, e.g., surfaces which have been polished or grinded in one direction. The surface anisotropy is usually characterized by a single number, the so-called Peklenik number $\gamma$, which is the ratio between the decay length $\xi_{x}$ and $\xi_{y}$ of the heightheight correlation function $\langle h(x, y) h(0,0)\rangle$ along the $x$ and $y$-directions, respectively, i.e. $\gamma=\xi_{x} / \xi_{y}$. Here it has been assumed that the $x$-axis is oriented along one of the principal directions of the anisotropic surface roughness.

Let us define the $2 \times 2$ matrix (we use polar coordinates so that the wave vector $\mathbf{q}=q(\cos \phi, \sin \phi))[13]$

$$
D(q)=\frac{\int \mathrm{d} \phi C(\mathbf{q}) \mathbf{q q} / q^{2}}{\int \mathrm{d} \phi C(\mathbf{q})}
$$

where the surface roughness power spectrum [14]

$$
C(\mathbf{q})=\frac{1}{(2 \pi)^{2}} \int \mathrm{d}^{2} x\langle h(\mathbf{x}) h(\mathbf{0})\rangle \mathrm{e}^{-i \mathbf{q} \cdot \mathbf{x}},
$$

where $\langle\ldots\rangle$ stands for ensemble average, and $h(\mathbf{x})$ is the height profile. For roughness with isotropic statistical properties, $C(\mathbf{q})$ will only depend on $q=|\mathbf{q}|$ and in this case $D(q)$ will be diagonal with $D_{11}=D_{22}=1 / 2$.

We will assume most of the time that $D(q)$ is independent of $q$ and in this case (1) is equivalent to

$$
D=\frac{\int \mathrm{d}^{2} q C(\mathbf{q}) \mathbf{q q} / q^{2}}{\int \mathrm{d}^{2} q C(\mathbf{q})} .
$$

In this case, in the coordinate system where $D$ is diagonal the flow conductivity matrix (defined below) $\sigma_{\text {eff }}$ will be diagonal too. Note that $\operatorname{Tr} D=D_{11}+D_{22}=1$, and in the coordinate system where $D$ is diagonal we can write $D_{11}=1 /(1+\gamma)$ and $D_{22}=\gamma /(1+\gamma)$, where $\gamma=\xi_{x} / \xi_{y}$ 
is the Peklenik number. Note that $D_{11}(1 / \gamma)=D_{22}(\gamma)$. If $D(q)$ (see (1)) depends on $q$ we may still define (in the coordinate system where $D(q)$ is diagonal) $\gamma=-1+1 / D_{11}$ as before, but the $x y$-coordinate system where $D(q)$ is diagonal may depend on $q$ (in which case the rotation angle, $\psi(q)$, of the $x$-axis relative to some fixed axis, is important information too, see ref. [13]). In this case $\gamma$ will depend on $q$ and we will refer to $\gamma(q)$ as the Peklenik function (and $\psi(q)$ as the Peklenik angle function). Note that since $D(q)$ is a symmetric tensor and since $\operatorname{Tr} D=$ 1 , the $D$-matrix has only two independent components. Thus, it is fully defined by the Peklenik function $\gamma(q)$ and the Peklenik angle function $\psi(q)$. In this paper we will assume that $\gamma(q)$ and $\psi(q)$ are constant.

\section{Fluid flow between solids with random surface roughness}

Consider two elastic solids with randomly rough surfaces. Even if the solids are squeezed in contact, because of the surface roughness there will in general be non-contact regions at the interface and, if the squeezing force is not too large, there will exist non-contact channels from one side to the other side of the nominal contact region. We consider now fluid flow at the interface between the solids. We assume that the fluid is Newtonian and that the fluid velocity field $\mathbf{v}(\mathbf{x}, t)$ satisfies the Navier-Stokes equation

$$
\frac{\partial \mathbf{v}}{\partial t}+\mathbf{v} \cdot \nabla \mathbf{v}=-\frac{1}{\rho} \nabla p+\nu \nabla^{2} \mathbf{v}
$$

where $\nu=\eta / \rho$ is the kinetic viscosity and $\rho$ is the mass density. For simplicity we will also assume an incompressible fluid so that

$$
\nabla \cdot \mathbf{v}=0
$$

We assume that the non-linear term $\mathbf{v} \cdot \nabla \mathbf{v}$ can be neglected (this corresponds to small inertia and small Reynolds number), which is usually the case in fluid flow between narrowly spaced solid walls. For simplicity we assume the lower solid to be rigid with a flat surface, while the upper solid is elastic with a rough surface, see fig. 1. We introduce a coordinate system $x y z$ with the $x y$-plane in the surface of the lower solid and the $z$-axis pointing towards the upper solid. Consider now squeezing the solids together in a fluid. Let $u(x, y, t)$ be the separation between the solid walls and assume that the slope $|\nabla u| \ll 1$. We also assume that $u / L \ll 1$, where $L$ is the linear size of the nominal contact region. In this case one expects that the fluid velocity varies slowly with the coordinates $x$ and $y$ as compared to the variation in the orthogonal direction $z$. Assuming also a slow time dependence, the Navier-Stokes equation is reduced to

$$
\eta \frac{\partial^{2} \mathbf{v}}{\partial z^{2}}=\nabla p
$$

Here and in what follows $\mathbf{v}=\left(v_{x}, v_{y}\right), \mathbf{x}=(x, y)$ and $\nabla=$ $\left(\partial_{x}, \partial_{y}\right)$ are two-dimensional vectors. Note that $v_{z} \approx 0$ and

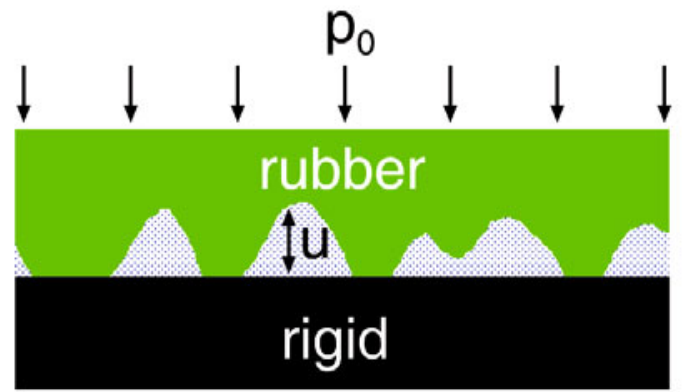

Fig. 1. An elastic solid (block) with a rough surface is squeezed (pressure $p_{0}$ ) in a fluid against a rigid solid (substrate) with a flat surface.

that $p(\mathbf{x})$ is independent of $z$ to a good approximation. From (4) one can obtain the fluid flow vector

$$
\mathbf{J}=-\frac{u^{3}(\mathbf{x})}{12 \eta} \nabla p .
$$

Assuming an incompressible fluid mass conservation demands that

$$
\frac{\partial u(\mathbf{x}, t)}{\partial t}+\nabla \cdot \mathbf{J}=0
$$

where the interfacial separation $u(\mathbf{x}, t)$ is the volume of fluid per unit area. In this last equation we have allowed for a slow time dependence of $u(\mathbf{x}, t)$ as would be the case, e.g., during fluid squeeze-out from the interfacial region between two solids.

The fluid flow at the interface between contacting solids with surface roughness on many length scales is a very complex problem, in particular at high squeezing pressures where a network of flow channels with rapidly varying width and height may prevail at the interface. This is illustrated in fig. 2, which shows the contact area (black) between two elastic solids with randomly rough surfaces. At high enough pressure the contact area will percolate, which will have a drastic influence on the interfacial fluid flow properties. Percolation corresponds to the moment when the narrowest channel disappears as a result of squeezing. It is also visible that for anisotropic roughness percolation occurs later in the direction of the roughness elongation (which is vertical in fig. 2).

Equations (5) and (6) describe the fluid flow at the interface between contacting solids with rough surfaces. One can show that after eliminating all the surface roughness components, the fluid current (given by (5)) takes the form

$$
\overline{\mathbf{J}}=-\sigma_{\mathrm{eff}} \nabla \bar{p}
$$

where $\bar{p}$ is the fluid pressure averaged over different realizations of the rough surface. The flow conductivity $\sigma_{\text {eff }}(\bar{u})$ is in general (for anisotropic surface roughness) a $2 \times 2$ matrix. The ensemble average of (6) gives

$$
\frac{\partial \bar{u}(\mathbf{x}, t)}{\partial t}+\nabla \cdot \overline{\mathbf{J}}=0
$$

Substituting (7) in (8) gives

$$
\frac{\partial \bar{u}(\mathbf{x}, t)}{\partial t}=\nabla \cdot\left(\sigma_{\mathrm{eff}} \nabla \bar{p}\right) .
$$




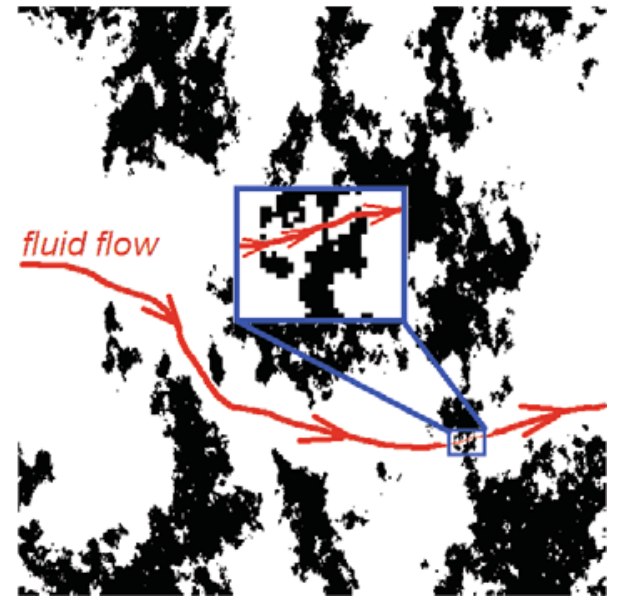

Fig. 2. (Color online) A snapshot of the contact before percolation in the $x$-direction (which is horizontal) for anisotropic roughness with Peklenik number 5/7. Red line indicates a fluid flow stream line. It is visible that fluid is able to flow from the left to the right part of the figure (or vice-versa) due to the presence of a narrow channel at some region of the contact. Inset presents the magnification of this region.

\section{Fluid flow conductivity $\sigma_{\text {eff }}$}

As was mentioned above, the fluid flow at the interface between contacting randomly rough surfaces requires taking into account the presence of the network of many interconnected flow channels. In a macroscopic approach this can be achieved through the use of the pressure flow factor. Here we have employed the 2D Bruggeman effective medium theory [15-18] to calculate (approximately) the pressure flow factor (see also appendix B).

For an anisotropic system, the effective medium flow conductivity $\sigma_{\text {eff }}$ is a $2 \times 2$ matrix. Let us introduce a $x y$ coordinate system and choose the $x$-axis along a principal axis of the $D$-matrix. In this case we can consider $\sigma_{\text {eff }}$ as a scalar which within the Bruggeman effective medium theory satisfies the relation:

$$
\frac{1}{\sigma_{\mathrm{eff}}}=\int \mathrm{d} u P(u) \frac{1+\gamma}{\gamma \sigma_{\mathrm{eff}}+\sigma(u)},
$$

where $P(u)$ is the probability distribution of interfacial separations, and where

$$
\sigma(u)=\frac{u^{3}}{12 \eta_{0}}
$$

Fluid flow along the $y$-axis is given by a similar equation with $\gamma$ replaced with $1 / \gamma$. The probability distribution $P(u)$ of interfacial separations has been derived in ref. [21]. Here we note that $P(u)$ has a delta function at the origin $u=0$ with the weight determined by the area of real contact:

$$
P(u)=\frac{A}{A_{0}} \delta(u)+P_{\mathrm{c}}(u),
$$

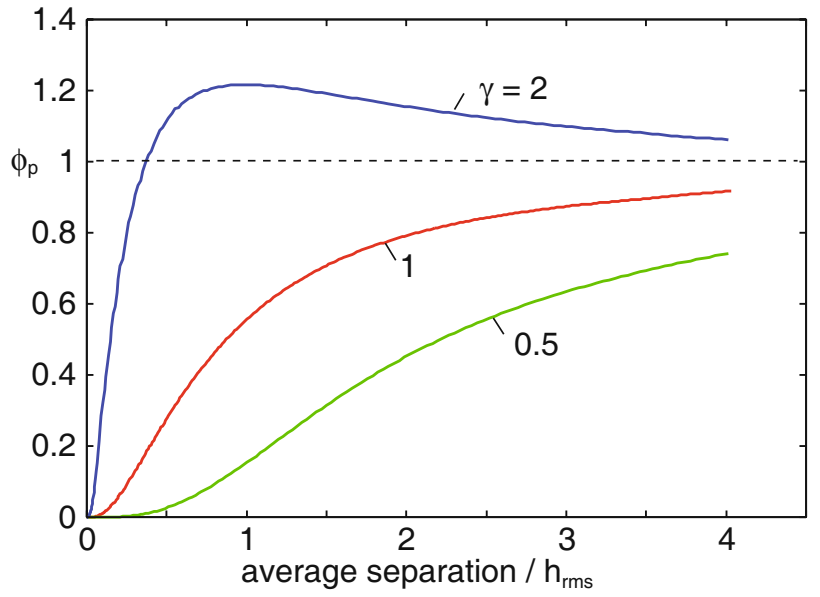

Fig. 3. (Color online) The pressure flow factor $\phi_{\mathrm{p}}$ as a function of the average interfacial separation $\bar{u}$, for anisotropic surfaces with the Peklenik numbers $\gamma=1 / 2,1$ and 2. In all cases the angular average power spectrum is of the type shown in fig. 4 with $H=0.9$ and the root-mean-square roughness $h_{\mathrm{rms}}=$ $10 \mu \mathrm{m}$.

where $P_{\mathrm{c}}(u)$ is a continuous (finite) function of $u$. Substituting this in (10) gives

$$
\frac{1}{\sigma_{\mathrm{eff}}}=\frac{A}{A_{0}} \frac{1+\gamma}{\gamma \sigma_{\mathrm{eff}}}+\int \mathrm{d} u P_{\mathrm{c}}(u) \frac{1+\gamma}{\gamma \sigma_{\mathrm{eff}}+\sigma(u)} .
$$

This equation is easy to solve by iteration.

In fig. 3 the pressure flow factor $\phi_{\mathrm{p}}=12 \eta_{0} \sigma_{\text {eff }} / \bar{u}^{3}$ as a function of the average interfacial separation $\bar{u}$ is displayed for anisotropic surfaces with the Peklenik numbers $\gamma=1 / 2,1$ and 2 (see also below). Note that $\phi_{\mathrm{p}}=0$ for $\bar{u}<\bar{u}_{\mathrm{c}}$, where $\bar{u}_{\mathrm{c}}$ is the average interfacial separation where the area of real contact percolates in the direction orthogonal to the fluid flow. In the Bruggeman effective medium theory this occurs when the area of real contact equals $A / A_{0}=\gamma /(\gamma+1)$. Thus for $\gamma=1 / 2,1$ and 2 the contact area percolates (so that no fluid flow occurs along the considered direction) when $A / A_{0}=1 / 3,1 / 2$ and $2 / 3$, respectively. This explains why $\phi_{\mathrm{p}}$ vanishes at much larger (average) interfacial separation (and hence smaller contact area) for $\gamma=1 / 2$ as compared to $\gamma=2$.

In obtaining the results presented below we have used the Persson contact mechanics theory for the contact area $A$ and the probability distribution $P(u)$ (see refs. [19-21]). This theory depends on the elastic energy $U_{\mathrm{el}}$ stored in the asperity contact regions and in this paper we use the simplest version for $U_{\text {el }}$ (see ref. [5]), where the $\gamma$-parameter (not the Peklenik number $)=1$. Comparison of the theory predictions with numerical simulations for small systems have shown that $\gamma \approx 0.45$ gives the best agreement between theory and the (numerical) experiments. However, using $\gamma=0.45$ (or $\gamma \neq 1$ in general) results in much longer computational time, with relatively small numerical changes as compared to using $\gamma=1$.

For large (average) surface separation $\bar{u}$ eqs. (5) and (6) can be solved exactly to leading order in $\left\langle h^{2}\right\rangle / \bar{u}^{2}$ (where $\left\langle h^{2}\right\rangle$ is the mean of the square of the surface rough- 
ness amplitude $h(x, y)$, where we have assumed $\langle h\rangle=$ 0) $[11,13]$

$$
\phi_{\mathrm{p}}=1+\frac{3\left\langle h^{2}\right\rangle}{\bar{u}^{2}}(1-3 D) .
$$

In appendix $\mathrm{A}$ we show that the Bruggeman effective medium theory gives the same expression for $\phi_{\mathrm{p}}$ to leading order in $\left\langle h^{2}\right\rangle / \bar{u}^{2}$ if we identify the $\gamma$-parameter in the effective medium theory with the Peklenik $\gamma$ defined by the $D$-matrix (see sect. 2). This result shows that the parameter $\gamma$ in the effective medium theory, which was introduced in a phenomenological way (as the ratio between the principle axis of an elliptic inclusion) in the effective medium theory (see ref. [13]), is indeed determined by the eigenvalues of the $D$-matrix as discussed in sect. 2 . This is a very important result and completes the theory for $\sigma_{\text {eff }}$ developed in ref. [13].

\section{Fluid squeeze-out}

Let us squeeze a rectangular rubber block (height $d$, width ( $x$-direction) $2 a$ and infinite length (y-direction)) against a substrate in a fluid. Assume that we can neglect the macroscopic deformations of the rubber block in response to the (macroscopically) non-uniform fluid pressure (which requires $d \ll a)[22,23]$. In this case $\bar{u}(\mathbf{x}, t)$ will only depend on time $t$. For this case from (9) we get

$$
\frac{\mathrm{d} \bar{u}}{\mathrm{~d} t}-\frac{\bar{u}^{3} \phi_{\mathrm{p}}(\bar{u})}{12 \eta} \frac{\partial^{2} \bar{p}}{\partial x^{2}}=0 .
$$

It follows from this equation above that the fluid pressure is parabolic

$$
\bar{p}(x, t)=\frac{3}{2} p_{\text {fluid }}(t)\left(1-\frac{x^{2}}{a^{2}}\right),
$$

where $2 a$ is the width of the contact region ( $x$-direction) and $p_{\text {fluid }}(t)$ the average fluid pressure in the nominal contact region. Combining the two equations above gives

$$
\frac{\mathrm{d} \bar{u}}{\mathrm{~d} t}=-\frac{\bar{u}^{3} \phi_{\mathrm{p}}(\bar{u})}{4 \eta a^{2}} p_{\text {fluid }}(t)
$$

If $p_{0}$ is the applied pressure acting on the top surface of the block, we have

$$
p_{\text {fluid }}(t)=p_{0}-p_{\text {cont }}(t),
$$

where $p_{\text {cont }}$ is the (locally, or ensemble averaged) asperity contact pressure. If the pressure $p_{0}$ is so small that for all times $\bar{u} \gg h_{\mathrm{rms}}$, then in this case $\phi_{\mathrm{p}}(\bar{u}) \approx 1$. For $\bar{u} \gg h_{\mathrm{rms}}$ we also have [7]

$$
p_{\text {cont }} \approx \beta E^{*} \exp \left(-\frac{\bar{u}}{u_{0}}\right),
$$

where $E^{*}=E /\left(1-\nu^{2}\right)$ (here $E$ is the Young's modulus and $\nu$ the Poisson ratio), and $u_{0}=h_{\mathrm{rms}} / \alpha$. The parameters $\alpha$ and $\beta$ depend on the fractal properties of the rough surface [7].

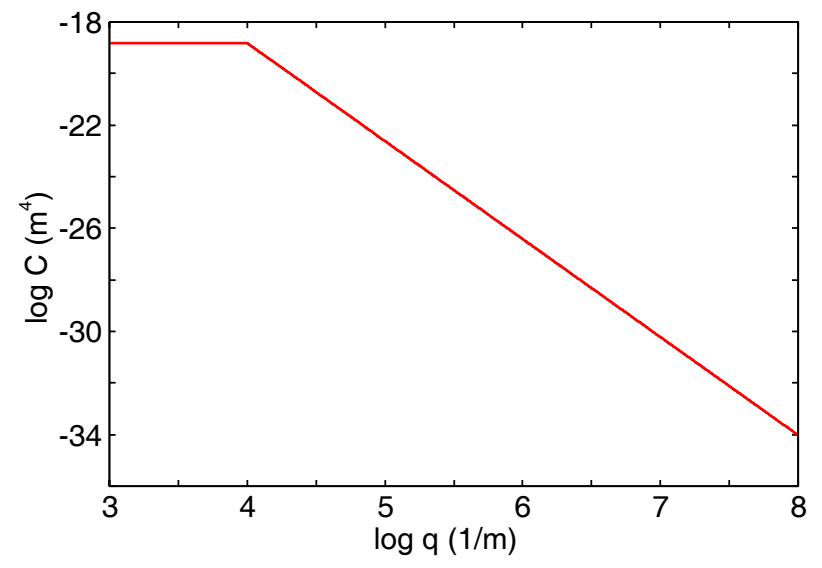

Fig. 4. (Color online) The logarithm (with 10 as basis) of angular average power spectrum as a function of the logarithm of the wave vector. For $q_{\mathrm{r}}<q<q_{1}$, with the roll-off wave vector $q_{\mathrm{r}}=10^{4} \mathrm{~m}^{-1}$ and the cut-off wave vector $q_{1}=10^{8} \mathrm{~m}^{-1}$, the surface is self-affine fractal with the Hurst exponent $H=0.9$. The low wave vector cut-off $q_{0}=10^{3} \mathrm{~m}^{-1}$ and $h_{\mathrm{rms}}=10 \mu \mathrm{m}$.

At high enough squeezing pressures and after long enough time, the interfacial separation will be smaller than $h_{\text {rms }}$, so that the asymptotic relation (16) will no longer hold. In this case the relation $p_{\text {cont }}(\bar{u})$ can be calculated using the equations given in ref. [8]. Substituting (15) in (14) and measuring pressure in unit of $p_{0}$, separation in unit of $h_{\mathrm{rms}}$ and time in unit of the relaxation time

$$
\tau=\frac{4 \eta a^{2} u_{0}}{h_{\mathrm{rms}}^{3} p_{0}}=\frac{4 \eta a^{2}}{\alpha h_{\mathrm{rms}}^{2} p_{0}},
$$

one obtains

$$
\frac{\mathrm{d} \bar{u}}{\mathrm{~d} t} \approx-\alpha^{-1} \phi_{\mathrm{p}}(\bar{u}) \bar{u}^{3}\left(1-p_{\text {cont }}\right),
$$

where $\alpha=h_{\text {rms }} / u_{0}$. In order to study the squeeze-out over a large time period, $t_{0}<t<t_{1}$, it is convenient to write $t=t_{0} e^{\mu}\left(0<\mu<\mu_{1}\right.$ with $\left.\mu_{1}=\ln \left(t_{1} / t_{0}\right)\right)$. In this case (18) takes the form

$$
\frac{\mathrm{d} \bar{u}}{\mathrm{~d} \mu} \approx-\alpha^{-1} t \phi_{\mathrm{p}}(\bar{u}) \bar{u}^{3}\left(1-p_{\text {cont }}\right) .
$$

This equation, together with the relation $p_{\text {cont }}(\bar{u})$, constitutes two equations for two unknowns $\left(\bar{u}\right.$ and $\left.p_{\text {cont }}\right)$ which can be easily solved by numerical integration.

We have studied the influence of percolation on the fluid squeeze-out for an elastic solid with randomly rough surface squeezed against a rigid flat surface in a fluid with the viscosity $\eta=12 \mathrm{Pas}$. In most of the studies the rough surface has the power spectrum shown in fig. 4 with the root-mean-square roughness $h_{\mathrm{rms}}=10 \mu \mathrm{m}$ and the large wave vector cut-off $q_{1}=10^{8} \mathrm{~m}^{-1}$. We also present some results for another surface with $q_{1}=10^{7} \mathrm{~m}^{-1}$. The elastic block has rectangular shape with the width $2 a=1.84 \mathrm{~cm}$ ( $x$-direction) and infinite length ( $y$-direction), and the squeezing pressure $p_{0}=2 \mathrm{MPa}$. The rubber has the Young's modulus $E=3 \mathrm{MPa}$ and Poisson ratio $\nu=0.5$. 


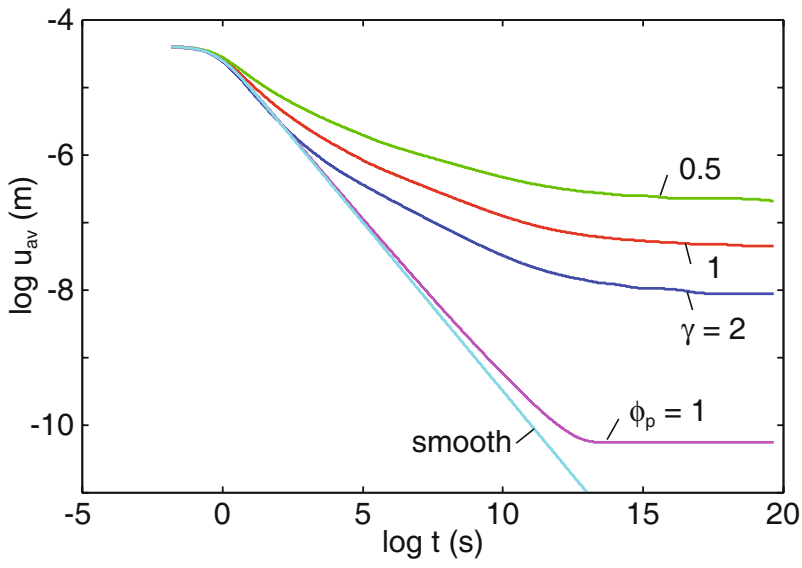

Fig. 5. (Color online) The logarithm (with 10 as basis) of the average surface separation as a function of the logarithm of the squeeze-out time. Calculations are for the Peklenik numbers $\gamma=0.5,1$ and 2 . The pink curve is the result with the fluid pressure flow factor $\phi_{\mathrm{p}}=1$. For a thin rectangular rubber block (width $2 a=1.84 \mathrm{~cm}$ ) with fractal-like surface roughness with the root-mean-square roughness amplitude $h_{\mathrm{rms}}=10 \mu \mathrm{m}$ and the large wave vector cut off $q_{1}=10^{8} \mathrm{~m}^{-1}$ (see fig. 4), squeezed against a flat rigid surface.

Figure 3 displays the pressure flow factor $\phi_{\mathrm{p}}$ as a function of the average interfacial separation $\bar{u}$, for anisotropic surfaces with the Peklenik numbers $\gamma=1 / 2,1$ and 2. Note that the flow factor and the viscosity $\eta$ enter the equation for the fluid squeeze-out as $\phi_{\mathrm{p}} / \eta$. Thus, $\phi_{\mathrm{p}}>1$ has the same effect as decreasing the viscosity and hence speedsup the squeeze-out. For $\gamma=2$ we have $\phi_{\mathrm{p}}>1$ except when the average interfacial separation $\bar{u}$ becomes so small that the contact area is close to the percolation threshold where $\phi_{\mathrm{p}}$ will vanish. Thus, at least for low squeezing pressures, where the interfacial separation never becomes so small that the area of real contact percolates, the fluid squeeze-out is enhanced by anisotropic roughness when the "groves" are in the direction of fluid flow. In a similar way, $\gamma<1$ is equivalent to increased viscosity, and slower fluid squeeze-out. However, these results are only valid for the line-contact geometry. For a circular or square contact area any roughness will speed up the squeeze-out. For an elliptic contact area with the groves oriented along one of the ellipse axis, the squeeze-out may be enhanced or slowed down depending on the ratio between the ellipse axis, and the value of the Peklenik number.

Figure 5 shows the logarithm of the average surface separation $\bar{u}$ as a function of the logarithm of the squeezeout time $t$ for the Peklenik numbers $\gamma=0.5,1$ and 2. Note that in all cases the squeeze-out time is the same but the final surface separation is the largest for $\gamma=0.5$. The reason is that for this $\gamma$ the contact area percolates (in the $x$ direction) for $A / A_{0}=1 / 3$, so that already when the area of real contact reaches this value fluid will be confined in the non-contact area and the rubber cannot come closer to the substrate as the fluid is essentially incompressible. For $\gamma=2$ the contact area percolates for $A / A_{0}=2 / 3$ which will occur at much smaller (average) surface separation

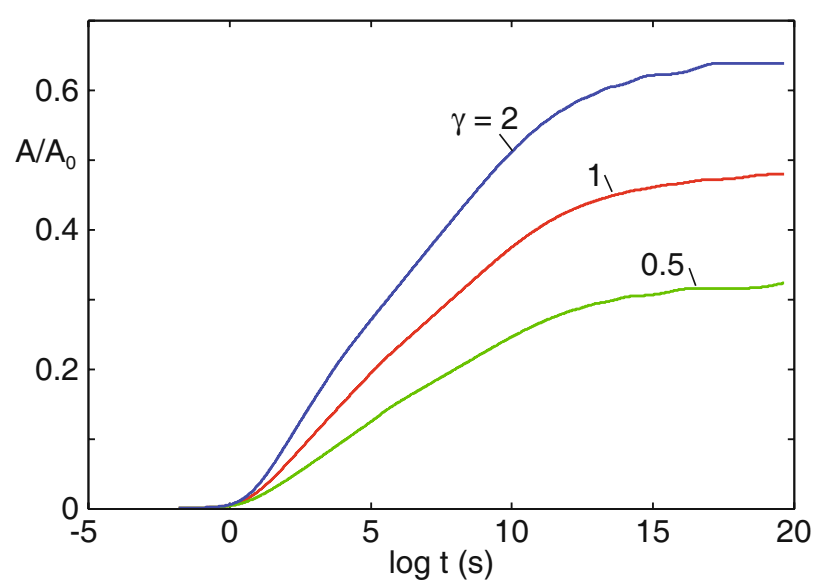

Fig. 6. (Color online) The relative area of real contact $A / A_{0}$ as a function of the logarithm (with 10 as basis) of the squeezeout time. Calculations are for the Peklenik numbers $\gamma=0.5$, 1 and 2 and the same parameters as in fig. 5 .

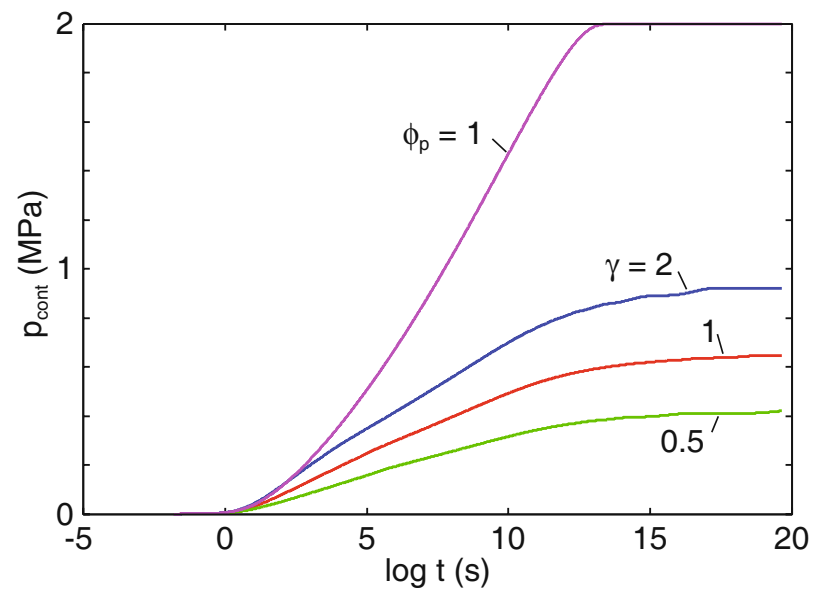

Fig. 7. (Color online) The nominal contact pressure $p_{\text {con }}$ as a function of the logarithm (with 10 as basis) of the squeeze-out time calculated for the Peklenik numbers $\gamma=0.5,1$ and 2 . The pink curve is the result with the fluid pressure flow factor $\phi_{\mathrm{p}}=1$.

than when $\gamma=1 / 2$. In fig. 5 we also show the average interfacial separation for perfectly smooth surfaces and for the case where $\phi_{\mathrm{p}}=1$ (which we refer to as the averageseparation theory [23]), where the influence of the surface roughness is not included in the fluid flow equation. In this case limiting (for large time) average separation $\bar{u}(\infty)$ is determined by the roughness alone independent of the fluid.

The time dependencies of the relative area of real contact $A / A_{0}$ for the same systems as studied in fig. 5 are presented in fig. 6 . For $\gamma=1 / 2,1$ and 2 the contact area saturates at $A / A_{0}=1 / 3,1 / 2$ and $2 / 3$ but the squeeze-out time is the same in all cases.

Figure 7 plots the nominal contact pressure $p_{\text {con }}$ as a function of the logarithm of the squeeze-out time for the same systems as studied in fig. 5. The applied squeezing pressure $p_{0}=2 \mathrm{MPa}$ is higher than the (nominal) contact pressure $p_{\text {con }}$ and the difference $p_{0}-p_{\text {con }}$ is carried by the 


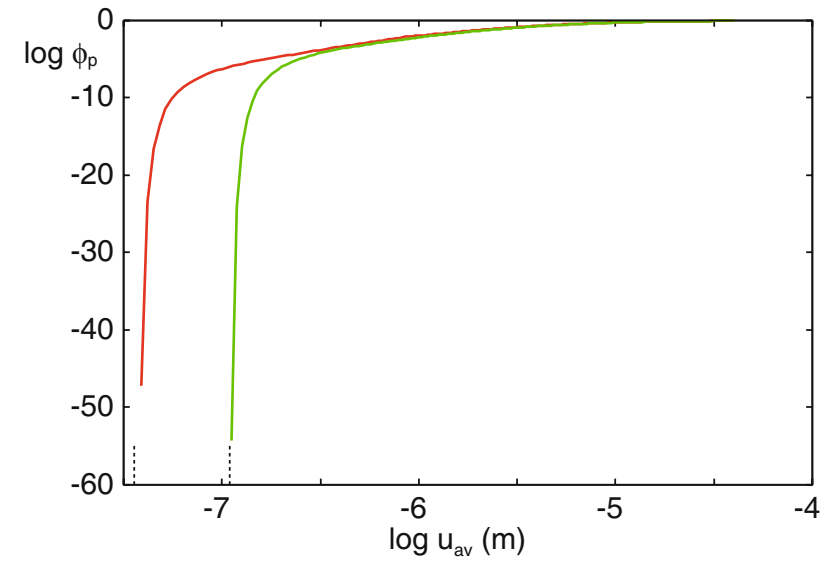

Fig. 8. (Color online) The logarithm (with 10 as basis) of the pressure flow factors $\phi_{\mathrm{p}}$ as a function of the logarithm of the average interfacial separation $\bar{u}$ for isotropic surfaces $(\gamma=1)$. In all the cases the angular average power spectrum is of the type shown in fig. 4 with $H=0.9$ and $h_{\mathrm{rms}}=10 \mu \mathrm{m}$ and with the cut-off wave vector $q_{1}=10^{8} \mathrm{~m}^{-1}$ (red curve) and $q_{1}=10^{7} \mathrm{~m}^{-1}$ (green curve).

fluid. Note that for $t>10^{17} \mathrm{~s}$ the contact pressure is constant but smaller than $p_{0}$ because some of the external load is carried by the (pressurized) fluid confined in the non-contact surface regions. The calculation with $\phi_{\mathrm{p}}=1$ does not account for the percolation of the contact area, so no pressurized confined fluid regions occur at the interface in this approximation, and after long enough time the full load is carried by the area of real contact. Note also that with $\phi_{\mathrm{p}}=1$ the squeeze-out occurs faster since the high resistance to fluid interfacial flow which occurs close to the percolation threshold (because of the narrow flow channels) is absent in this approximation.

Let us now study the influence of changes in the surface roughness power spectra on the squeeze-out. Figure 8 displays the logarithm of the pressure flow factors $\phi_{\mathrm{p}}$ as a function of the logarithm of the average interfacial separation $\bar{u}$, for a surface with isotropic roughness (Peklenik number $\gamma=1$ ) for two values of the cut-off wave vector $q_{1}=10^{8} \mathrm{~m}^{-1}$ (red curve) and $q_{1}=10^{7} \mathrm{~m}^{-1}$ (green curve) in the power spectrum. Note that removing one decade of the shortest wavelength roughness has no influence on the pressure flow factor for large interfacial separation because this region of fluid squeeze-out is dominated by the long-wavelength large-amplitude roughness components. However, the shortest-wavelength roughness is very important for small interfacial surface separations (which require high squeezing pressures and long enough contact time). Thus, removing short wavelength roughness moves the surface-area percolation threshold to larger interfacial separation.

The dependence of the logarithm of the average surface separation on the logarithm of the squeeze-out time for the two surfaces studied in fig. 8 is shown in fig. 9. Note that including more short wavelength surface roughness results after a long contact time in a smaller (average) surface separation, which is expected as more fluid flow channels

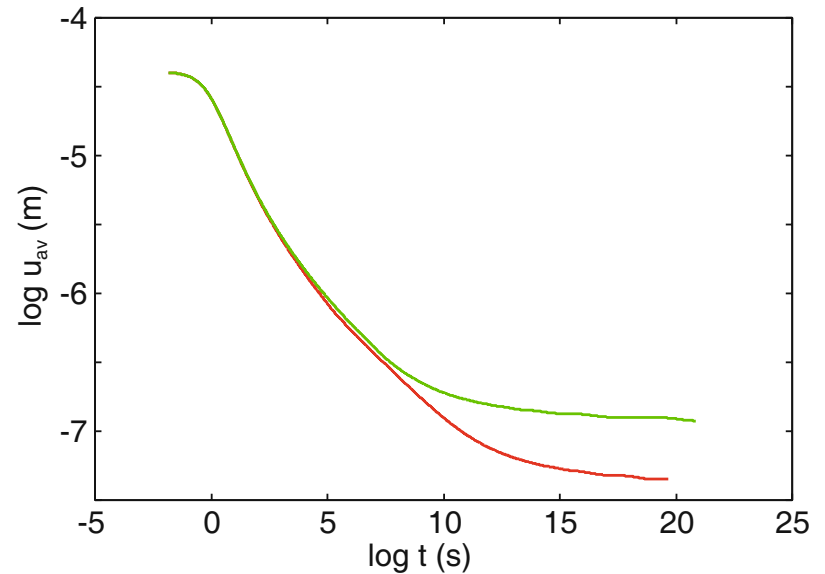

Fig. 9. (Color online) The logarithm (with 10 as basis) of the average surface separation as a function of the logarithm of the squeeze-out time. Calculations are for the Peklenik number $\gamma=$ 1. For a thin rectangular rubber block (width $2 a=1.84 \mathrm{~cm}$ ) with fractal-like surface roughness with the root-mean-square roughness amplitude $h_{\mathrm{rms}}=10 \mu \mathrm{m}$ and the large wave vector cut off $q_{1}=10^{8} \mathrm{~m}^{-1}$ (see fig. 4) (lower (red) curve) and $q_{1}=$ $10^{7} \mathrm{~m}^{-1}$ (upper (green) curve), squeezed against a flat rigid surface.

(which can be observed only at high magnification) exist in this case. However, for "short" time the squeeze-out is identical for both surfaces, as is indeed expected because in this region of "large" surface separation the squeeze-out is dominated by the long wavelength roughness, which is the same in both cases.

\section{Computer simulation of percolation}

After the description of the theoretical approach, let us present the numerical simulation technique and results. Classical MD simulations have been carried out to verify the Bruggeman effective medium theory prediction that the contact area percolates when $A / A_{0}=\gamma /(1+\gamma)$.

The model consists of an elastic block with a flat bottom surface which is brought into contact with a randomly rough rigid substrate. The latter (see fig. 10) contains $N_{x} \times N_{y}=512 \times 512$ atoms which occupy the sites of a square lattice in the $x y$-plane with the lattice constant of $a=2.6 \AA$. Self-affine fractal topography with Hurst exponent value of $H=0.8$ and the power spectrum analogous to the displayed in fig. 4 have been used. The isotropic randomly rough surface profile of the substrate was obtained using the procedure described in ref. [14], based on the adding plane waves with random phases. The anisotropic roughness was generated by stretching the isotropic one in one direction (corresponding to the vertical or $y$-direction in the contact pictures below) accordingly to the specified value of the Peklenik number. In the present study the values of Peklenik number equal to $1.0,5 / 6,5 / 7,5 / 8$ and $1 / 2$ are used.

When two elastic solids with rough surfaces come into contact, the elastic deformations perpendicular to the con- 


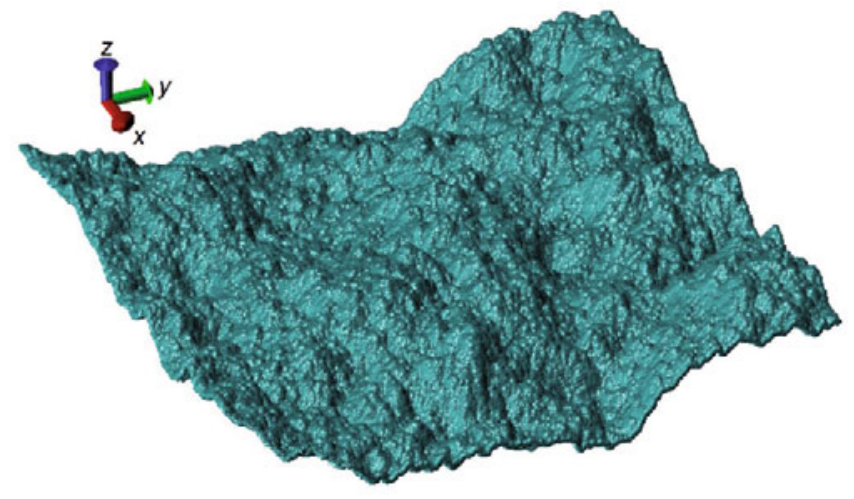

Fig. 10. (Color online) Randomly rough substrate with the $h_{\mathrm{rms}}=10 \AA$ used in the MD simulations. In the figure atoms have rescaled $z$-coordinates with the factor of 5 . The roughness is isotropic, i.e. Peklenik number $\gamma=1.0$. The snapshot is produced with Visual Molecular Dynamics software [24].

tacting plane extend into the solids a characteristic length $\lambda$ that could be as large as the contacting plane's lateral size $L$. Therefore, in order to properly capture the mechanical response of the solids within the contact region, the elastic properties of the material have to be considered up to a distance $L$ in the normal direction to the contacting plane. In our model this is achieved through the use of a "smart-block" coarse-graining approach [25], which allows to drastically accelerate computations of a contact mechanics problem by reducing the number of dynamical variables without significant loss of accuracy.

In the original implementation [25] smart-block atoms are placed on the sites of a simple cubic lattice, and coupled with springs to their nearest neighbors. Full resolution down to the atomic level is maintained at the interface. The coarse graining is applied to the upper layers starting from the third one from the bottom (first) layer, and it involves merging together a box made of $m_{x} \times m_{y} \times m_{z}$ particles. The three integers $m_{x}, m_{y}$ and $m_{z}$ are called merging factors along the three axes. The equilibrium position of each new "super-particle" is in the center of mass of the group of particles merged together. Its mass is $m_{x} m_{y} m_{z}$ times the mass of the original particles, so that the density does not change. Along the direction of merging the new spring constants for elongation and bending are redefined to guarantee identical elastic response to the original fully atomistic block.

In our model, the bottom layer of the elastic smartblock (or simply block for brevity) consists of an array of $512 \times 512$ atoms which form a simple square lattice with lattice constant $a=2.6 \AA$. Periodic boundary conditions are applied in the $x y$-plane. The mass of a block atom is $197 \mathrm{amu}$, the Young modulus of the block is $E=250 \mathrm{GPa}$ and its Poisson ratio is $\nu=1 / 4$. Smart-block thickness is equal to $1350.7 \AA$, which is slightly larger than its lateral dimensions $L_{x}=L_{y}=N_{x} a=1331.2 \AA$. The elastic slab consists of 12 atomic layers, and merging factors of 2 (in all 3 directions) are used for all layers, except the 6 'th and the 11 'th. The block contains 615780 atoms, and the total number of atoms involved in the simulations is 877924 .
The atoms at the block-substrate interface interact via a repulsive potential $U(r)=4 \varepsilon\left(r_{0} / r\right)^{12}$, where $r$ is the interatomic distance and the parameter $\varepsilon$ corresponds to the binding energy between two atoms at the separation $r=2^{1 / 6} r_{0}$. In our calculations the values $r_{0}=3.28 \AA$ and $\varepsilon=18.6 \mathrm{meV}$ are used. Zero temperature is maintained during the simulations using a Langevin thermostat [26] which allows to exclude the influence of the thermal noise on the results, and the equations of motion have been integrated using Verlet's method $[26,27]$ with a time step of $\Delta t=1 \mathrm{fs}$. In the present study the squeezing process proceeds as follows. The upper surface of the smart-block is moved towards the substrate at a constant velocity of $v=5 \mathrm{~m} / \mathrm{s}$ with the block being compressed as its bottom layer approaches the substrate.

We have checked that the level of coarse-graining does lead to converged results, i.e., making a slower coarsegraining as one proceeds into the block away from the interface results in negligible changes in the results. The elastic waves emitted from the interface are damped out by small viscous damping acting on the "atoms" in the block. We have also checked that this damping and the speed with which the solids are brought together are both small enough not to influence the results presented below.

In the context of the present study, the most important output from the simulations is the ratio $A / A_{0}$ of the real contact area $A$ to the apparent one $A_{0}$, and, in particular, that value $A_{\text {perc }} / A_{0}$ at which percolation occurs. We note that there is no unique way how to define contact at the atomistic level [28], but this is not important in the present context as we are only interested in the contact area at the point where the contact area percolates, and this quantity is not sensible to the way the contact area is defined (see below). The quantity $A / A_{0}$ is defined using a procedure analogous to the calculation of the probability distribution of interfacial separations [21]. For each substrate atom the distance along the $z$-direction to the neighboring block atom is computed, and if this distance is less than some specific value $h_{0}$, the substrate atom is marked and considered to be in contact with the block. As was ascertained in ref. [21], $h_{0} \approx 4 \AA$ for the mentioned parameters of the interatomic potential, and in the current calculations we also used the values of $5 \AA$ and $6 \AA$ for comparison. The ratio $A / A_{0}$ is computed by dividing the number of atoms being in contact to the total number of substrate atoms.

The moment of percolation of the contact area is defined using visual inspection of contact pictures, some of which are shown in figs. 2, 11 and 12, where a pixel corresponds to a substrate atom. Black pixels reflect atoms in contact, while white pixels correspond to atoms located farther than $4 \AA$ from the corresponding nearest block atoms. Figure 11 presents the evolution of the contact area of the block squeezed against a randomly rough substrate with isotropic statistical properties (Peklenik number $\gamma=1$ ). The contact area percolates (along both the $x$ - and $y$-directions) for $A / A_{0} \approx 0.48$. In fig. 12 similar pictures for a substrate with anisotropic statistical properties (Peklenik number $\gamma=1 / 2$ ) are displayed. In this 


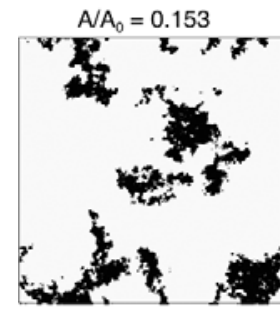

$A / A_{0}=0.476$
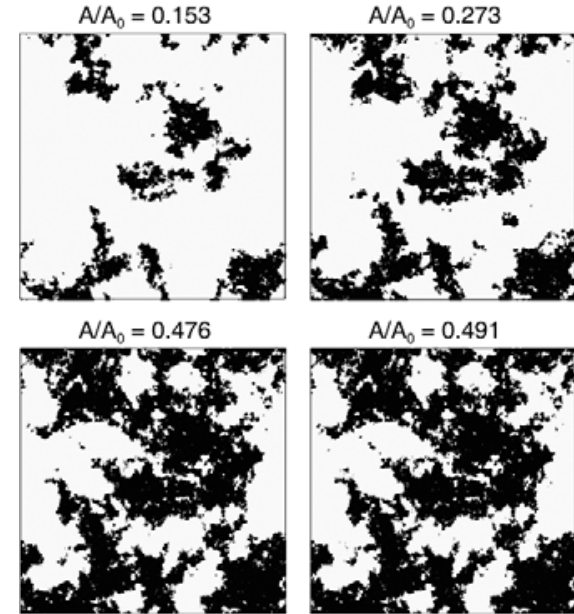

$\mathrm{A} / \mathrm{A}_{0}=0.491$
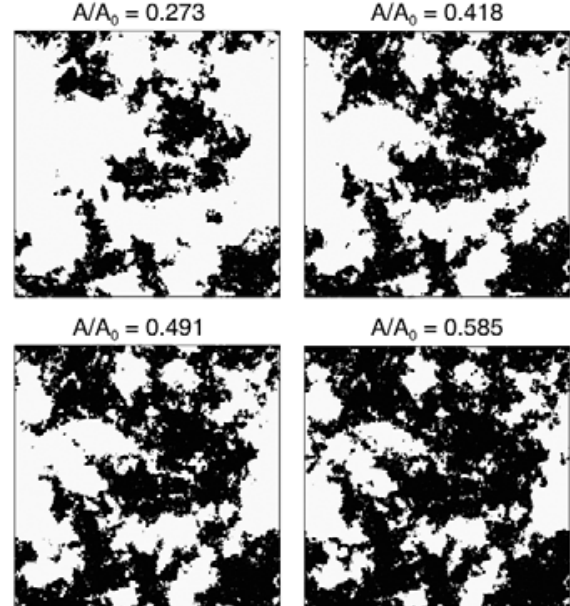

$A / A_{0}=0.585$

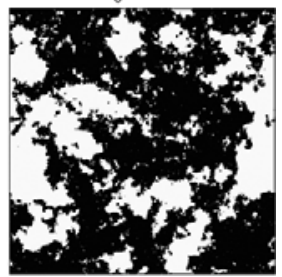

Fig. 11. Contact pictures for an elastic block with a flat surface squeezed against a hard, randomly rough surface with isotropic statistical properties (Peklenik number $\gamma=1$ ). The contact area percolates (along both the $x$ and $y$-directions) for $A / A_{0} \approx$ 0.48

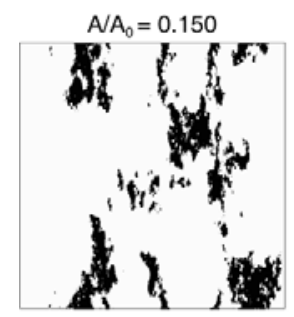

$A A_{0}=0.451$

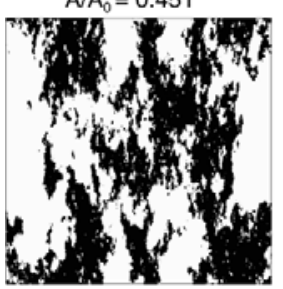

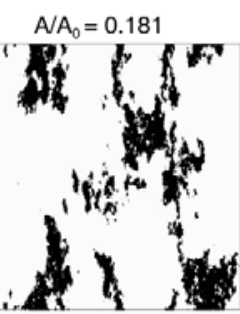

$A / A_{0}=0.539$

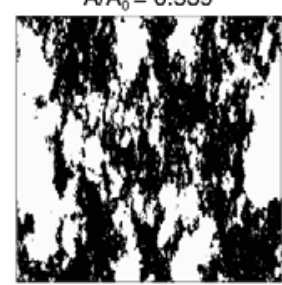

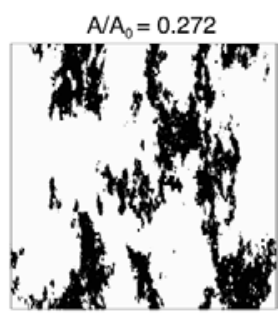

$A / A_{0}=0.583$

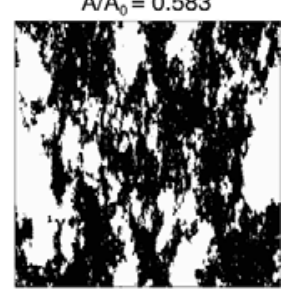

Fig. 12. Contact pictures for an elastic block with a flat surface squeezed against a hard, randomly rough surface with anisotropic statistical properties (Peklenik number $\gamma=1 / 2$ ). The contact area percolates in the $x$-direction when $A / A_{0} \approx$ 0.27 and in the $y$-direction when $A / A_{0} \approx 0.54$.

case the contact area percolates in the $x$-direction when $A / A_{0} \approx 0.27$ and in the $y$-direction when $A / A_{0} \approx 0.54$.

In fig. 13 we show the results for the (relative) contact area at percolation, $A_{\text {perc }} / A_{0}$, as a function of the Peklenik number $\gamma$. All the values of $h_{0}$ give the similar trends. However, on average percolation occurs for larger value of $A / A_{0}$ when bigger $h_{0}$ is used. Note the relatively large spread in the results of the simulations, and that in the numerical experiments the percolation tends to occur at slightly smaller contact area than predicted by the effective medium theory. We believe that both effects are mainly due to the small size of the considered simulation cell, while the theory is for an infinite system. Accordingly to experimental $[29,30]$ and theoretical investigations [31-34], finite size effects can be observed in

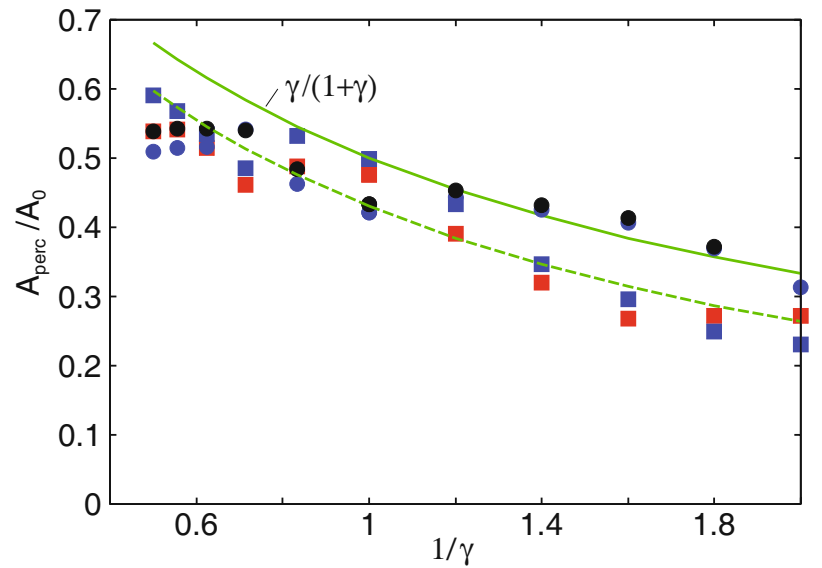

Fig. 13. (Color online) The relative contact area at percolation, $A_{\text {perc }} / A_{0}$, as a function of the Peklenik number $\gamma$. The square and circular symbols are obtained from two numerical simulations using surfaces generated with different seeds for the random number generator. The solid line is the prediction of the Bruggeman effective medium theory and the dashed line is the solid line shifted by 0.07 towards lower relative contact area. Contact between the solids is assumed to occur when the interfacial separation becomes smaller than $4 \AA$ (red symbols), $5 \AA$ (blue symbols) and $6 \AA$ (black symbols).

systems which have properties dependent on the percolation of a quantity, defined by a geometrical combination of small constituent blocks. These effects are usually manifested in two ways. First, there may be oscillations of the measured quantities [31], such as density of clusters. It is expected that such oscillations reflect the fluctuations in the distribution of the size of constituent blocks in the finite system. They appear whenever the average is taken over a finite number of samples, and decay as this number is increased. The second finite size effect is a shift of the percolation threshold average $[29,32,33]$, and it was shown that for a smaller system percolation occurs earlier than for a larger one. Therefore, data scattering in fig. 13 can be attributed to the first type of finite size effects, which is caused by fluctuations in the topography of the substrate from sample to sample. A small discrepancy between numerical and analytical results may be due to the second type of the finite size effects.

\section{Experimental investigation of percolation}

The Bruggeman effective medium theory predicts that for randomly rough surfaces, with isotropic statistical properties, the contact area percolates at $A / A_{0}=0.5$. In an effort to directly measure the contact area at a fixed (but relevant) magnification, we have performed experiments using an instrumented cantilever based load platform with an in situ microscope that images through a polished glass disk. This apparatus is described in more detail in ref. [35]. Schematically this setup is shown in fig. 14. The rough elastomeric block is loaded against the smooth (nearly perfectly smooth in comparison) glass surface and a digital 


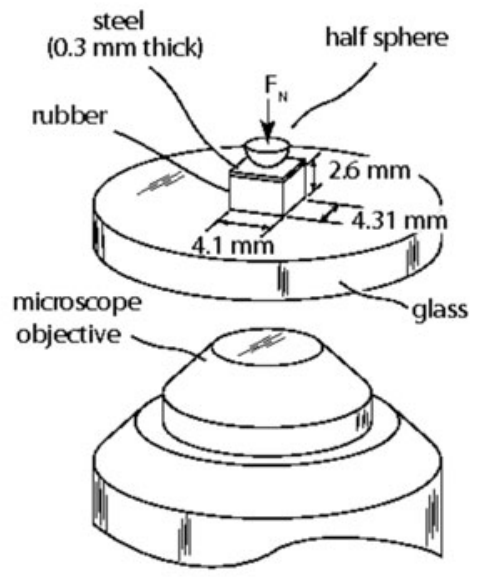

Fig. 14. A schematic of the optical in situ loading experiment. A randomly rough elastomer sample is loaded against a polished glass window. Directly beneath the contact is a $10 \times$ microscope objective focused on the contacting surface of the glass window. The rubber is backed by a stainless steel plate. External load is transmitted from a hemispherical probe through the steel back plate to insure a uniform, momentfree pressure distribution across the glass-rubber contact. The spherical probe is attached to a cantilever based force transducer instrumented by a capacitance probe to resolve externally applied load $\left(F_{\mathrm{N}}\right)$ and tip displacement.

image of the contact is collected through the microscope system, which is built below the contact.

The randomly rough elastomeric sample (filled natural rubber) has a measured apparent area of contact that is $4.3 \mathrm{~mm} \times 4.1 \mathrm{~mm}$, and a thickness of $2.6 \mathrm{~mm}$. The smooth side of the elastomeric sample was adhered to $0.3 \mathrm{~mm}$ thick stainless-steel backing plate that is used to transmit the normal load from a spherical pin. This produces a nearly uniform pressure and moment free pressure distribution across the contact area, because the point contact on the stainless steel plate is located at the centroid. The cantilever based force transducer uses capacitive probes that have been shown to provide experimental uncertainties in force of better than $1 \mathrm{mN}$. The loading assembly is attached to a piezoelectric positioning stage, and all of the loading phases of the experiment were performed by ramping the piezo at $1 \mu \mathrm{m} / \mathrm{s}$ (the actual rate of deformation of the contact is the difference between the loading displacement and the cantilever deflection).

The microscope objective was centered directly below the rubber sample and the optical axis was aligned normal to the polished glass surface; the focal plane of the image was at the contacting surface of the glass disk. The particular objective used for these experiments was $10 \times$, and when coupled to the 5 megapixel detector gives a size of each pixel at $\sim 350 \mathrm{~nm} \times 350 \mathrm{~nm}$. This imaging procedure reveals areas that are in contact (at this magnification) as dark, and areas that are not in contact as bright. Interestingly, the illumination scheme also produces a long range interference pattern for those areas that are within approximately $5 \mu \mathrm{m}$ of separation. Figure 15a) shows a rep- a)

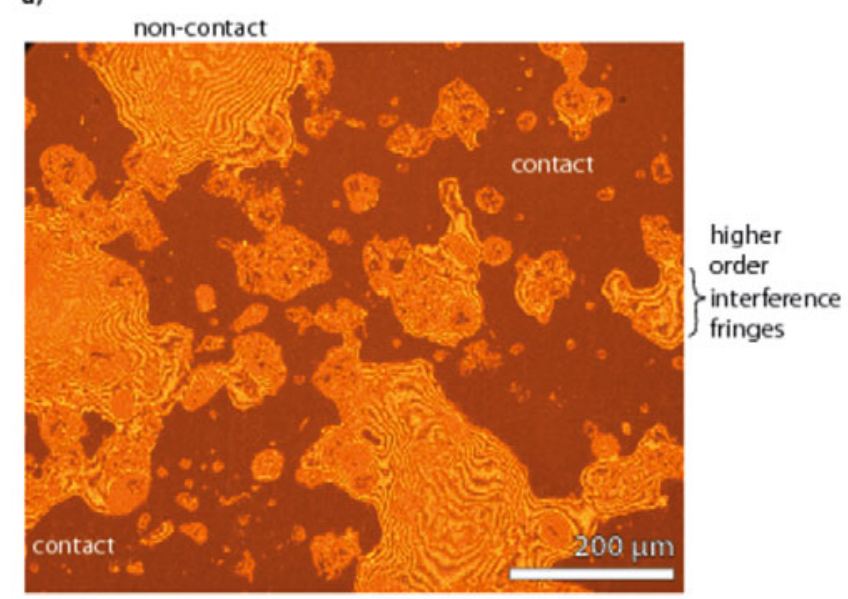

b)
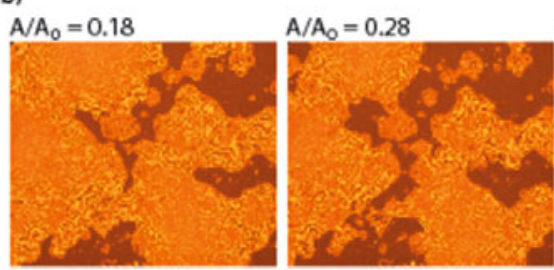

$A / A_{0}=0.50$
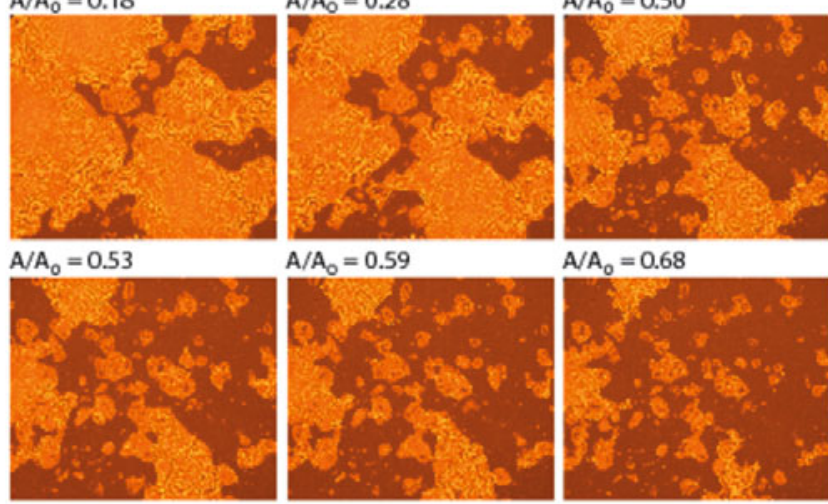

$A / A_{0}=0.68$

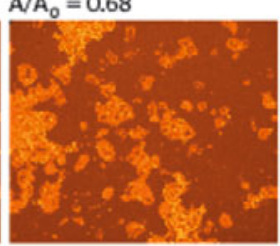

Fig. 15. a) An example image of the contacting surfaces where the dark areas represent contact, the lighter areas representing non-contact and the higher order interference fringes are caused by constructive and destructive interference of light reflected from the glass and rubber surfaces. b) Contact pictures for the randomly rough elastomer squeezed against the flat, hard, polished glass disc for various $A / A_{0}$.

resentative image during contact with appropriate scale bars, and the various regions identified.

A pattern of contacting areas during a loading experiment is show in fig. 15b). During this experiment the ratio of the real area of contact to apparent area of contact varied from approximately 0.2 to 0.9 . Note that, in accordance with the theory, the area of real contact percolate at or very close to $A / A_{0}=0.5$. The externally applied loads reach approximately $7 \mathrm{~N}$, where the adhesion force and dead weight load were not measured. The loading profile contained periods of loading ramps and stationary holds. Each image from the experiment was analyzed using an imaging processing algorithm [35], and the ratio of the real area of contact to the apparent area of contact is plotted versus the externally applied load in fig. 16 .

We have studied the surface topography of the rubber surface using AFM and line-scan methods. From the height profile we have calculated the surface roughness power spectrum. In fig. 17 we show the logarithm of the surface roughness power spectrum as a function of the 


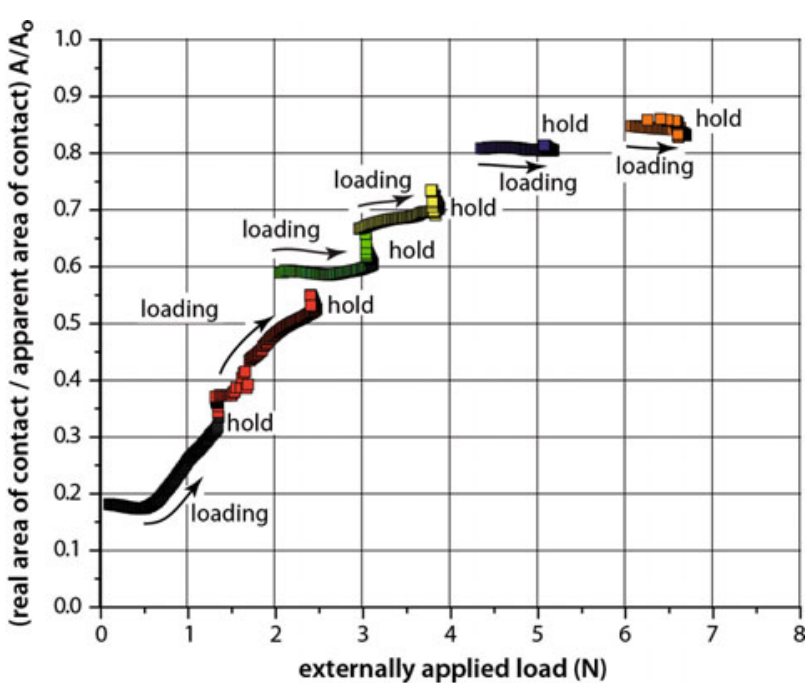

Fig. 16. (Color online) The ratio of the real area of contact to the apparent area of contact (relative area of contact, $A / A_{0}$ ) is plotted versus the externally applied load. Each data point represents a processed image. During loading the piezo stage (which is mounted to the force transducer/loading assembly) was ramped at $1 \mu \mathrm{m} / \mathrm{s}$ (the actual rate of deformation of the contact is the difference between the loading displacement and the cantilever deflection). After each loading phase there was a hold phase which allowed for relaxation of the contact for approximately 30 seconds. This loading and hold sequence was repeated until the relative area of contact steadied at a value near 0.85 .

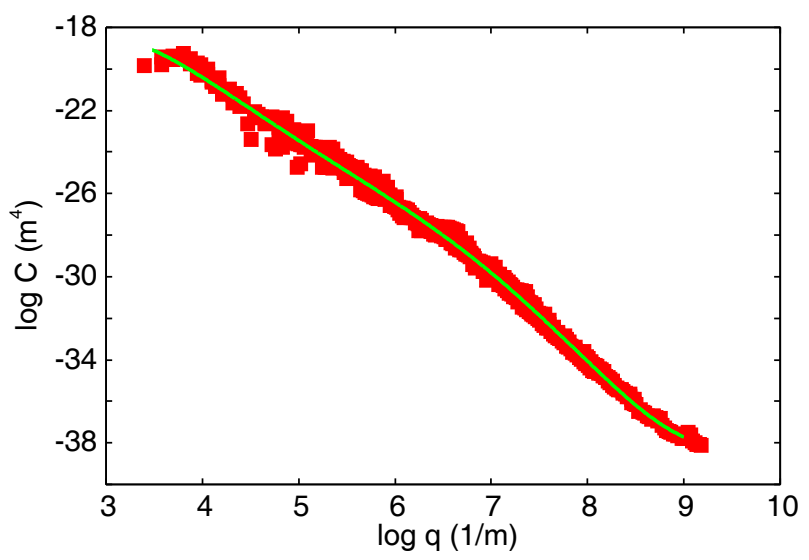

Fig. 17. (Color online) The logarithm (with 10 as basis) of the surface roughness power spectrum as a function of the logarithm of the wave vector for the rubber surface used in the study reported on in fig. 16. The green line is a fit to the experimental data and is the power spectrum of a surface with the root-mean-square (rms) roughness amplitude $h_{\mathrm{rms}}=2.65 \mu \mathrm{m}$ and rms slope 0.47 and total surface area $A_{\mathrm{tot}}=1.2 A_{0}$.

logarithm of the wave vector for the rubber surface used in the study reported on in fig. 16. The green line is a fit to the experimental data and corresponds to surface with the root-mean-square (rms) roughness amplitude $h_{\mathrm{rms}}=2.65 \mu \mathrm{m}$ and rms slope 0.47 and total surface area $A_{\text {tot }}=1.2 A_{0}$.

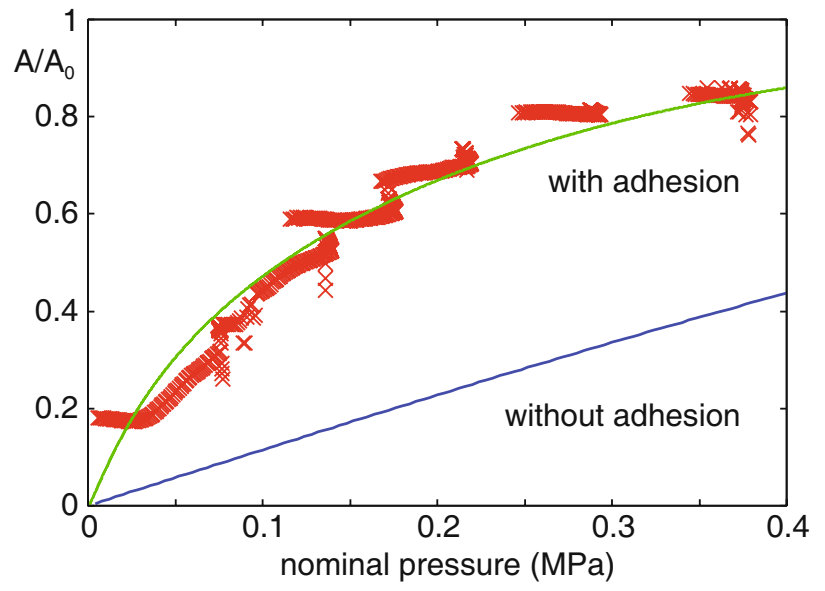

Fig. 18. (Color online) The normalized contact area $A / A_{0}$ as a function of the squeezing pressure $p_{0}$. The red symbols are the experimental data from fig. 16 and the green and blue solid lines the theory prediction using the Persson contact mechanics theory (in vacuum) including adhesion and without adhesion, respectively.

In fig. 18 we compare the measured relation between $A / A_{0}$ and the nominal squeezing pressure $p_{0}=F / A_{0}$ with the prediction of the Persson contact mechanics theory including adhesion (green curve) [36] and without adhesion (blue curve) [19]. In the calculation we have used the power spectrum shown in fig. 17 and the elastic modulus $E=4.5 \mathrm{MPa}$ (the shear modulus was measured in $10 \%$ strain to be $1.5 \mathrm{MPa}$ and we assume the Poisson ratio $\nu=0.5)$. In the calculation including adhesion we have used the work of adhesion $\gamma_{0}=0.08 \mathrm{~J} / \mathrm{m}^{2}$ which is close to what is expected for (filled) natural rubber in contact with glass for a very slowly closing crack. Good agreement prevails when adhesion is included in the analysis, while neglecting the adhesion results in too small contact area.

For $A / A_{0}>0.5$ one would perhaps expect some difference between the experiment and the theory since the air in the non-contact regions will be sealed off (trapped) from the surrounding atmosphere when the contact area percolates. Thus, as the squeezing pressure increases, the air in the sealed non-contact region will be compressed and carry some part of the external load. The theory has been developed for contact in vacuum, but it would in principle be easy to extend the theory to include (in a mean-field way) the influence of trapped gas on the contact mechanics, but this is beyond the present study. However, the influence of the trapped air seams anyway very small in the present case, maybe it has enough time to diffuse into the rubber. If the experiment is repeated in a low-viscosity fluid (in order for fluid squeeze-out to have a negligible influence on the experiment) one would expect the contact area to saturate at the percolation value $A / A_{0} \approx 0.5$, because the contact pressure (in the present experiment involving rubber) is too small to compress the fluid. We plan to perform experiments for rubber in contact with a flat glass surface in water to test this theory prediction.

In fig. 19 we show the calculated normalized contact area $A / A_{0}$ and the normalized effective interfacial bind- 


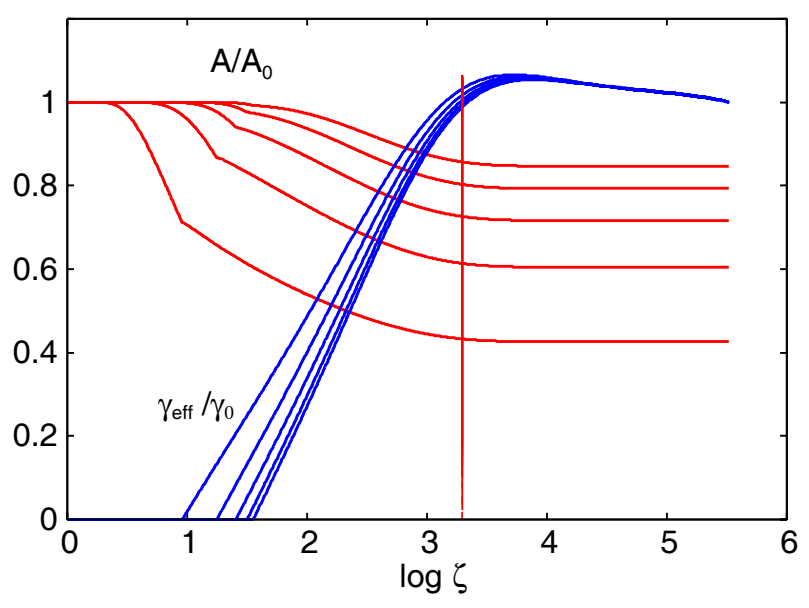

Fig. 19. (Color online) The calculated normalized contact area $A / A_{0}$ (red lines) and the normalized effective interfacial binding energy (per unit area) $\gamma_{\text {eff }} / \gamma_{0}$ (blue lines) as a function of the logarithm (with 10 as basis) of the magnification. Results are shown for several squeezing pressure $p_{0} / p_{\max }=0.2,0.4$, $0.6,0.8$ and 1 , where $p_{\max }=0.4 \mathrm{MPa}$ is the maximal squeezing pressures. The vertical line denotes the magnification $\zeta_{\exp }$ corresponding to the resolution of the experiments.

ing energy (per unit area) $\gamma_{\text {eff }} / \gamma_{0}$ as a function of the logarithm of the magnification. Results are shown for several squeezing pressures $p_{0} / p_{\max }=0.2,0.4,0.6,0.8$ and 1 , where $p_{\max }=0.4 \mathrm{MPa}$ is the maximal squeezing pressures. The vertical line shows the magnification $\zeta_{\exp }$ $\left(\zeta_{\exp } q_{0}=2 \pi / \lambda_{\exp }\right.$ where $\lambda_{\exp }$ is the experimental resolution of order $\sim 1 \mu \mathrm{m}$ ) at which the experiment reported on above was performed. Note that already at this magnification the contact area has almost reached its plateau value, and increasing the magnification does only result in a very small change in the contact area. This is a result of the adhesion, which pulls the surfaces into nearly complete contact in the asperity contact regions observed at the magnification $\zeta_{\text {exp }}$. In contrast, in the absence of adhesion the (apparent) contact area decreases monotonically with increasing magnification.

\section{Application to prefillable syringes}

Syringes are the most common prefilled parenteral drug delivery systems, in which a rubber plunger stopper is actionated to expel the injectable liquid drug from a cylindrical glass or polymer barrel (see fig. 20). Low, uniform and stable gliding forces values of the plunger stopper are critical to patient comfort and healthcare professional convenience in manual use of prefillable syringes; when syringes are used in conjunctions with self-administration devices (autoinjectors) gliding performance is even more critical [37]. The plunger stopper need to ensure also the container integrity or sealing, to ensure no drug dose changes and maintain sterility. This is particularly important for the prefilled syringes where the drug can be stored in the device for several years before use. Traditionally the inner surface of the syringe is lubricated by a high viscosity

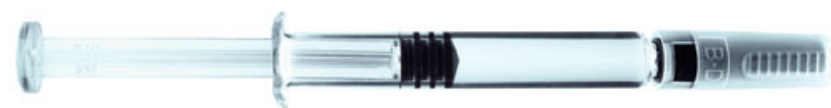

Fig. 20. A syringe consists of a cylindrical glass or polymer barrel with a rubber plunger stopper with ribs to seal the fluid.

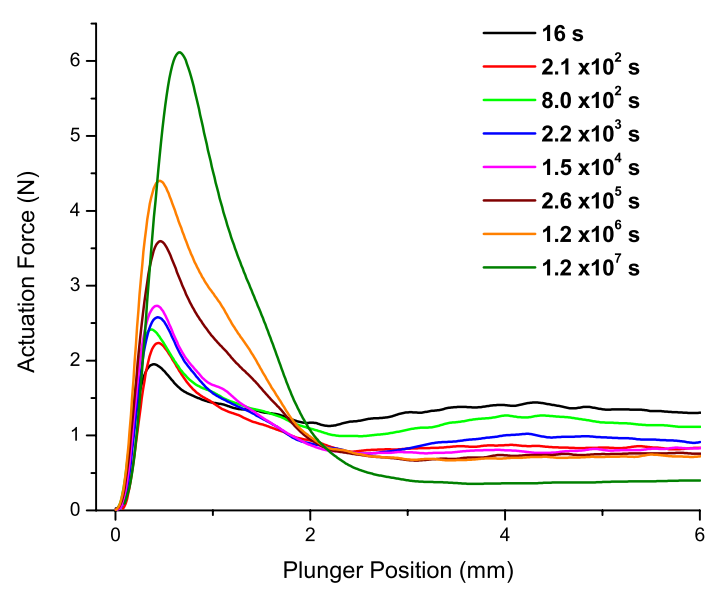

Fig. 21. (Color online) The force as a function of displacement of the rubber stopper for various time of stationary contact. Note that the breakloose force increases with the time of stationary contact due to squeeze-out of the high viscosity lubricant fluid (silicon oil). Note also that the kinetic friction force decreases with the time of stationary contact which probably is due to viscoelastic relaxation of the rubber which lowers the contact pressure and changes the shape of the rubber rib contact region.

silicone oil (viscosity $\eta \approx 0.35-10 \mathrm{~Pa}$ s) to ensure good device's gliding performance. The nominal contact pressure between the ribs of the stopper and the glass barrel is a critical parameter for sealing and reaches very high values, of order $\sim 1 \mathrm{MPa}$. Nevertheless, the silicon oil is squeezed out from the glass-barrel interface very slowly because of its high viscosity. This squeeze-out causes a continuous increase of the static friction before a syringe is used, as shown in fig. 21. In some cases this may result in such a high breakloose force that hinders the device functionality during the injection. It is therefore of great importance to understand the exact origin and nature of the breakloose friction.

In the application to syringes, the system consists of round or flat ribs in contact with the syringe's barrel surface. The contact can be described as a rectangular region of width $w \approx 1 \mathrm{~mm}$ ( $x$-direction) and of infinite length (because of the circular nature of the contact region) (ydirection). In the present study we will assume that the Hertzian contact formed at the interface between the ribs and the barrel is nominally flat, i.e., we neglect the macroscopic curvature of the barrels and the ribs, and the deformations of the rubber ribs which result from the fluid pressure buildup which is highest at the center of the contact region. The latter results in a bending of the bottom 


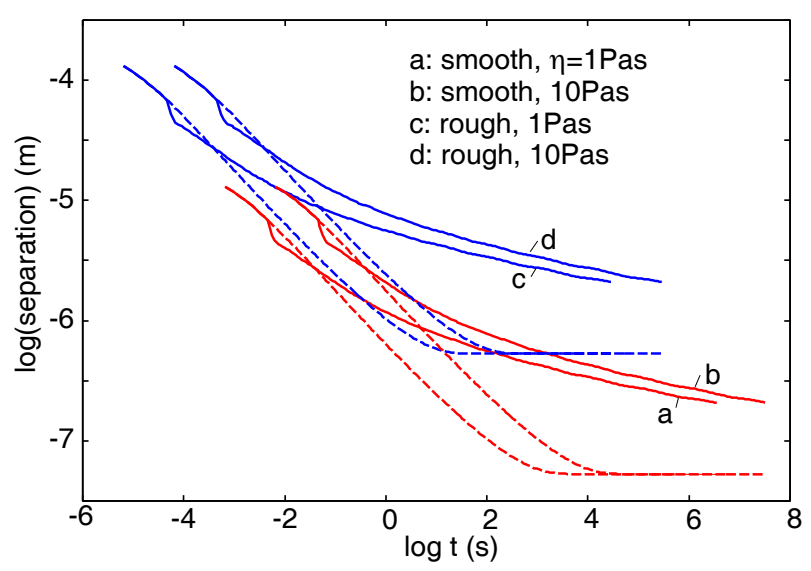

Fig. 22. (Color online) The logarithm (with 10 as basis) of the (average) separation between the surfaces as a function of the logarithm of time. For a rectangular contact region of width $w=0.7 \mathrm{~mm}$ and for the smooth and the rough surfaces. The nominal squeezing pressure $p=1 \mathrm{MPa}$ and the rubber elastic modulus $E=3 \mathrm{MPa}$. The solid lines are obtained using the full theory (effective medium theory for the fluid pressure flow factor) while the dashed line is obtained using the averageseparation theory (where the pressure flow factor is unity). Results are shown for the "smooth" and "rough" surfaces (see text), and for fluid viscosity $\eta=1$ and 10 Pas.

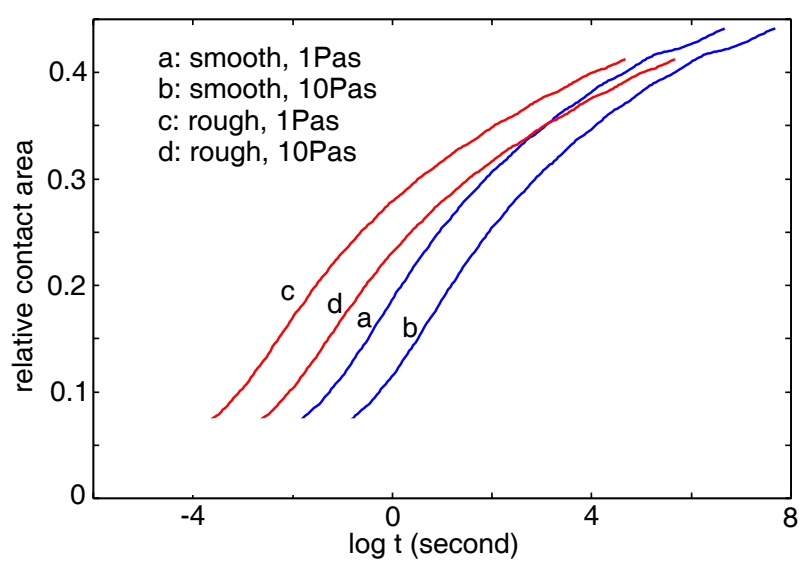

Fig. 23. (Color online) The relative contact area $A / A_{0}$ (where $A_{0}$ is the nominal contact area) as a function of the logarithm (with 10 as basis) of time. For a rectangular contact region of width $w=0.7 \mathrm{~mm}$ and for the smooth and the rough surfaces. The nominal squeezing pressure $p=1 \mathrm{MPa}$ and the rubber elastic modulus $E=3 \mathrm{MPa}$. The results have been obtained using the full theory (effective medium theory for the fluid pressure flow factor).

surface of the rubber cylinder inwards at the center, giving rise to slower squeeze-out. Thus, the squeeze-out problem we study will result in a lower bound for the actual squeeze-out time.

The assumption that the surfaces in the contact region are flat and parallel implies that the separation $\bar{u}(t)$ is a function only of time. For this case, for the cylinder geometry (rectangular contact region) we have derived the basic equations determining $\bar{u}(t)$ in sect. 5 .
We have performed calculations for two different values of fluid viscosity, namely $\eta=1$ and $10 \mathrm{Pas}$ (as is typical for the silicon oils used to lubricate the surfaces) and for two surface roughness cases which we refer to as smooth and rough. The surface power spectra of both surfaces correspond to self-affine fractal surfaces with the fractal dimension $D_{\mathrm{f}}=2$ as measured for the rubber stopper surfaces. The surface roughness power spectra had a similar form as in fig. 4 but with different slope and with $q_{0}=q_{\mathrm{r}}$ and $q_{1}=3 \times 10^{8} \mathrm{~m}^{-1}$. For the rough surface $q_{0}=3 \times 10^{3}$ and the root-mean-square roughness $50 \mu \mathrm{m}$ while for the smooth surface $q_{0}=3 \times 10^{4}$ and the root-mean-square roughness $5 \mu \mathrm{m}$. The calculated power spectrum for most rubber stoppers was very similar to what we used in our model calculations.

Figure 22 plots the logarithm of the (average) separation between the surfaces as a function of the logarithm of time. The results are for a nominal contact area with the width $w=0.7 \mathrm{~mm}$ squeezed against the smooth and the rough surfaces. The nominal squeezing pressure $p=1 \mathrm{MPa}$ and the rubber elastic modulus $E=3 \mathrm{MPa}$. The solid lines are obtained using the full theory (effective medium theory for the fluid pressure flow factor) while the dashed line is produced using the average-separation theory (where the pressure flow factor is unity) [23]. Note the slow squeeze-out: even after $\sim 10^{8} \mathrm{~s}$ (about 3 years) the fluid film thickness is decreasing with time. Complete squeeze-out would result in the separation indicated by the dashed lines for large time and will never occur for the rough surfaces due to confinement (trapping) of fluid at sealed-off regions on the surface. That is, some "cavities" will be filled with fluid which cannot leak-out due to the "walls" resulting from the rubber-substrate contact.

In fig. 23 we show the relative contact area $A / A_{0}$ (where $A_{0}$ is the nominal contact area) as a function of the logarithm of time. The results have been obtained using the full theory (effective medium theory for the fluid pressure flow factor).

The start-up or breakloose friction will depend sensitively on the squeeze-out dynamics and in a first approximation (at least for small start-up velocities) one may assume that it is proportional to the area of real contact shown in fig. 23.

We have measured the start up (or breakloose) friction force and the kinetic friction force as a function of the logarithm of the waiting time for glass syringes, see figs. 21 and 24. To generate these data, syringes were filled with water, and the syringe's barrel-rubber stopper system were assembled as follows: (a) for short storage times $(<100 \mathrm{~s})$ the systems were assembled manually, and (b) for long storage time all systems were assembled in a vacuum placement unit. At different time points, 5 syringes were actionated in an automatic compression bench at fixed speed, the force needed to initiate and sustain the movement of the plunger were recorded.

It is interesting to note that as the time approaches zero the start-up friction values approach the kinetic friction values (not shown). This is of course expected because 


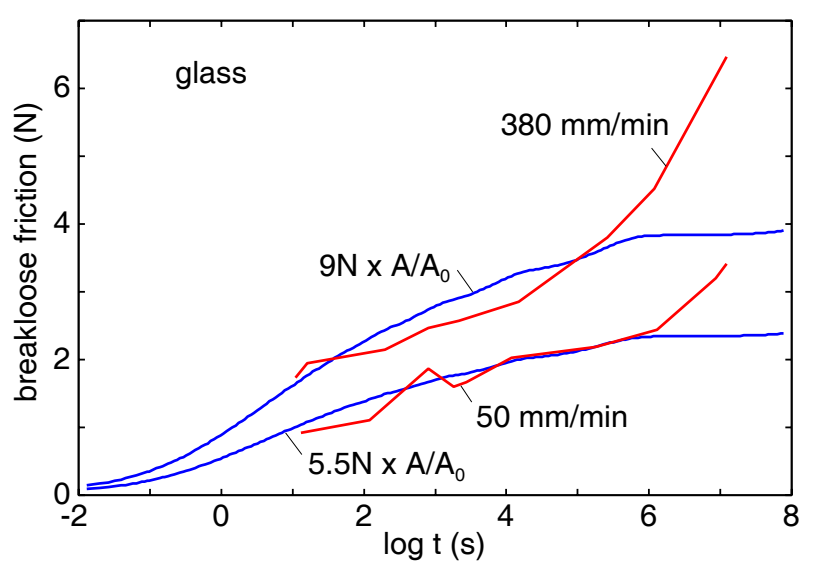

Fig. 24. (Color online) Red curves: The measured static (or breakloose) friction force as a function of the logarithm (with 10 as basis) of the waiting time for syringes with glass tube and for driving velocities $50 \mathrm{~mm} / \mathrm{min}$ and $380 \mathrm{~mm} / \mathrm{min}$. Blue curves: the calculated relative contact area $A / A_{0}$ times $5.5 \mathrm{~N}$ (lower curve), and times $9 \mathrm{~N}$ (upper curve) as a function of the waiting time.

after a very short waiting time the fluid film thickness will be similar to during the insertion move.

Figure 24 shows the measured static (or breakloose) friction force as a function of the logarithm of the waiting time for syringes with glass tube and driving velocities $v=380 \mathrm{~mm} / \mathrm{min}$ and $50 \mathrm{~mm} / \mathrm{min}$ (red curves). The blue curves are the calculated relative contact area $A / A_{0}$ (from fig. 23 , smooth, $\eta=10$ Pas) times 9 Newton (upper curve) and times 5.5 Newton (lower curve), as a function of the waiting time. The prefactors $9 \mathrm{~N}$ and $5.5 \mathrm{~N}$ were chosen to get a good agreement with the measured data for $t<$ $10^{6} \mathrm{~s}$. Assuming that the friction force is proportional to the contact area $A$, we can write

$$
F_{\mathrm{s}}(t)=\sigma_{\mathrm{f}} A(t)=\sigma_{\mathrm{f}} A_{0} \times\left(A(t) / A_{0}\right),
$$

where $\sigma_{\mathrm{f}}$ is the frictional shear stress in the area of real contact. Thus we have $\sigma_{\mathrm{f}} A_{0} \approx 9 \mathrm{~N}$ for $v=380 \mathrm{~mm} / \mathrm{min}$ and $\sigma_{\mathrm{f}} A_{0} \approx 5.5 \mathrm{~N}$ for $v=50 \mathrm{~mm} / \mathrm{min}$. The nominal contact area is estimated as $A_{0}=3 \times 2 \pi R w$ where $R \approx 3.5 \mathrm{~mm}$ is the radius and $w \approx 1 \mathrm{~mm}$ the width of the nominal rubber-glass (circular) contact region. Thus, $A_{0} \approx 6.6 \times 10^{-5} \mathrm{~m}^{2}$ giving $\sigma_{\mathrm{f}} \approx 1.36 \times 10^{5} \mathrm{~Pa}$ at $v=$ $380 \mathrm{~mm} / \mathrm{min}$ and $\sigma_{\mathrm{f}} \approx 0.83 \times 10^{5} \mathrm{~Pa}$ at $v=50 \mathrm{~mm} / \mathrm{min}$. This is in good agreement with the measured frictional shear stress of surfaces lubricated by long-chain silicon oil at contact pressures of order $\sim 1 \mathrm{MPa}$. Thus, frictional shear stress studies of confined fluids (including high-viscosity silicon oil) using the Surface Forces Apparatus (SFA) [38,39], and other experiments [40], and MD calculations [41], at nominal pressures of order a few MPa, resulting in a (nearly) universal expression for the effective viscosity $[38,39,41]$

$$
\eta_{\mathrm{eff}} \approx B \dot{\gamma}^{-n}
$$

where, if $\eta_{\text {eff }}$ is measured in Pas, $B \approx 5 \times 10^{4}$ and $n \approx 0.9$. The shear rate $\dot{\gamma}=v / d$, where $v$ is the sliding velocity and $d$ the film thickness. Thus the frictional shear stress

$$
\sigma_{\mathrm{f}}=\eta_{\mathrm{eff}} \dot{\gamma} \approx B \dot{\gamma}^{0.1} \approx B(v / d)^{0.1} .
$$

According to Surface Forces Apparatus (SFA) measurements (see refs. $[38,39]$ ) at the contact pressure $\sim 1 \mathrm{MPa}$ the trapped silicon oil film is of order $d \approx 3 \mathrm{~nm}$. Using the equation above gives $\sigma_{\mathrm{f}} \approx 2.1 \times 10^{5} \mathrm{~Pa}$ for $v=$ $380 \mathrm{~mm} / \mathrm{min}$ and $\sigma_{\mathrm{f}} \approx 1.7 \times 10^{5} \mathrm{~Pa}$ for $v=50 \mathrm{~mm} / \mathrm{min}$. The calculated frictional shear stresses $\sigma_{\mathrm{f}}$ are remarkable close to what we deduce from the experimental data for the glass-rubber case. The ratio between the calculated shear stresses $9 / 5.5 \approx 1.6$ is larger than expected from the theory $(380 / 50)^{0.1} \approx 1.22$. We note that in the SFA measurements $[38,39]$, at the nominal squeezing pressures $2.2 \mathrm{MPa}$ and $3.7 \mathrm{MPa}$ the thickness of the trapped silicon oil film was 2.8 and $2.0 \mathrm{~nm}$ (corresponding to 4 and 3 monolayers), respectively. In the syringe applications the nominal pressure is a little smaller (about $1 \mathrm{MPa}$ ) and one may therefore expect a slightly thicker film (maybe 5 monolayers rather than 4 or 3 ) and perhaps slightly smaller shear stress than calculated above, which would be in accordance with the observations above.

The measured breakloose friction force does not exhibit a saturation for $t>10^{6} \mathrm{~s}$ as expected if the timedependence of the friction just depends on the timedependence of the area of real contact. Thus, for $t>10^{6} \mathrm{~s}$ the friction in fact increases faster than at earlier times, see fig. 24. This indicates that not only the contact area, but also the frictional shear stress $\sigma_{\mathrm{f}}$ in the area of real contact, increases with time, at least for very large time. This may be due to a dewetting transition in the area of real contact or due to a decrease in the thickness of the trapped silicon oil film, say from 4 to 3 monolayers. Such layering transitions have been studied both theoretically and experimentally [42], and are thermally activated process and may be characterized by very long relaxation times if the barrier for the nucleation of the squeeze-out of a monolayer is high enough.

In the experiments it was also observed that the sustaining or kinetic friction forces decreased continuously with the time of stationary contact which we attribute to viscoelastic relaxation in the rubber which reduces the contact pressure. This "softening" of the rubber with increasing time will also influence the the time-evolution of the area of real contact.

\section{Summary and conclusion}

In the present work, a phenomenological theory for the fluid flow at the interface between contacting randomly rough surfaces with anisotropic statistical properties has been described. The theory utilizes the concept of the pressure flow factor, and it has been shown that this quantity significantly depends on the statistical properties of the rough surface, characterized by the Peklenik number $\gamma$. The developed theory also allowed to analyze the influence of the roughness anisotropy on the evolution of the average surface separation, relative area of contact and 
the nominal contact pressure during squeezing of a rubber block with rough surface against a substrate in a fluid. The influence of changes in the surface roughness power spectrum on squeezing has also been revealed. The theory predictions for the percolation threshold of the contact area have been verified using MD simulations and squeezing experiments. The MD results show reasonably good agreement with the Bruggeman effective medium theory prediction $A / A_{0}=\gamma /(1+\gamma)$. The experiments for the isotropic surfaces corroborate theoretical results for the percolation threshold, and also indicate that after the percolation is reached the trapped air may influence the squeezing process. This phenomenon requires further investigations, where numerical and experimental techniques can be employed to study the squeeze-out of the considered system in a fluid. Finally, we have considered fluid squeeze-out in the context of prefilled syringes, where removal of the lubricant fluid between the ribs of the rubber plunger stopper and the glass barrel, results in a breakloose (or static) friction which increases continuously with the time of stationary contact. We have shown that this process is quite complex and may involve thermally activated events (e.g., the nucleation of squeeze-out of monolayer films or dewetting processes), characterized by very long (of order several month) relaxation times. This topic requires further studies where the interface is probed at the nanometer scale.

This work, as part of the European Science Foundation EUROCORES Program FANAS, was supported from funds by the DFG and the EC Sixth Framework Program, under contract N ERAS-CT-2003-980409.

\section{Appendix A. Effective medium theory}

According to the effective medium theory, the effective flow conductivity $\sigma_{\text {eff }}$ is determined by

$$
\left\langle\frac{(1+\gamma) \sigma_{\mathrm{eff}}}{\gamma \sigma_{\mathrm{eff}}+\sigma(u)}\right\rangle=1,
$$

where $\sigma(u)=u^{3} /(12 \eta)$, where $u=u(\mathbf{x})$ is the interfacial separation. We have defined

$$
\langle f(u)\rangle=\int \mathrm{d} u P(u) f(u),
$$

where $P(u)$ is the distribution of interfacial separations. Let $\bar{u}=\langle u\rangle$ be the average interfacial separation. We write $u=\bar{u}+\delta u$ and $\bar{\sigma}=\bar{u}^{3} /(12 \eta)$. Thus, we get

$$
\begin{aligned}
\frac{\sigma-\bar{\sigma}}{\bar{\sigma}} & =\frac{\delta \sigma}{\bar{\sigma}}=\frac{(\bar{u}+\delta u)^{3}-\bar{u}^{3}}{\bar{u}^{3}} \\
& =3 \epsilon+3 \epsilon^{2}+\epsilon^{3},
\end{aligned}
$$

where $\epsilon=\delta u / \bar{u}$. We can rewrite (A.1) as

$$
\frac{\bar{\sigma}}{\sigma_{\text {eff }}}=(1+\gamma)\left\langle\left(\gamma \frac{\sigma_{\text {eff }}}{\bar{\sigma}}+1+\frac{\delta \sigma}{\bar{\sigma}}\right)^{-1}\right\rangle .
$$

If we introduce $y=\sigma_{\text {eff }} / \bar{\sigma}$ and use (A.2), we get

$$
\frac{1}{y}=(1+\gamma)\left\langle\frac{1}{\gamma y+1+3 \epsilon+3 \epsilon^{2}+\epsilon^{3}}\right\rangle
$$

or

$$
\frac{1}{y}=\frac{1+\gamma}{\gamma y+1}\left\langle\frac{1}{1+\frac{3 \epsilon+3 \epsilon^{2}+\epsilon^{3}}{\gamma y+1}}\right\rangle .
$$

Thus

$$
\frac{1}{y}+\gamma=(1+\gamma)\left\langle\frac{1}{1+\frac{3 \epsilon+3 \epsilon^{2}+\epsilon^{3}}{\gamma y+1}}\right\rangle .
$$

We assume $\epsilon=\delta u / \bar{u} \ll 1$. When $\epsilon=0$ we have $y=1$. For nonzero but small $\epsilon$ we write $y=1+\delta$ so that $1 / y \approx 1-\delta$. Thus, to lowest order in $\epsilon$ we can write (A.4) as

$$
\begin{aligned}
1+\gamma-\delta & =(1+\gamma)\left\langle\frac{1}{1+\frac{3 \epsilon+3 \epsilon^{2}+\epsilon^{3}}{\gamma+1}}\right\rangle \\
& =(1+\gamma)\left\langle 1-\frac{3 \epsilon+3 \epsilon^{2}+\epsilon^{3}}{\gamma+1}+\left(\frac{3 \epsilon}{1+\gamma}\right)^{2}\right\rangle \\
& =\frac{3(\gamma-2)}{1+\gamma}\left\langle\epsilon^{2}\right\rangle .
\end{aligned}
$$

Thus, to first order in $\left\langle\epsilon^{2}\right\rangle$

$$
y=1+\frac{3(\gamma-2)}{1+\gamma}\left\langle\epsilon^{2}\right\rangle
$$

or

$$
\sigma_{\mathrm{eff}}=\bar{\sigma}\left(1+\frac{3(\gamma-2)}{1+\gamma} \frac{\left\langle\delta u^{2}\right\rangle}{\bar{u}^{2}}\right) .
$$

Now, for large separation $\left\langle\delta u^{2}\right\rangle=\left\langle h^{2}\right\rangle=h_{\text {rms }}^{2}$. Thus, in this case

$$
\sigma_{\mathrm{eff}}=\bar{\sigma}\left(1+\frac{3(\gamma-2)}{1+\gamma} \frac{\left\langle h^{2}\right\rangle}{\bar{u}^{2}}\right) .
$$

However, this limiting case can be studied directly from Reynolds equation using perturbation theory which gives $[11,13]$

$$
\sigma_{\mathrm{eff}}=\bar{\sigma}\left(1+\frac{3\left\langle h^{2}\right\rangle}{\bar{u}^{2}}(1-3 D)\right),
$$

where

$$
D=\frac{\int \mathrm{d}^{2} q C(\mathbf{q}) \mathbf{q q} / q^{2}}{\int \mathrm{d}^{2} q C(\mathbf{q})} .
$$

Note that $\operatorname{Tr} D=D_{11}+D_{22}=1$. In a coordinate system where $D$ is diagonal, we write $D_{11}=1 /(1+\gamma)$ and $D_{22}=\gamma /(1+\gamma)$. Substituting $D=1 /(1+\gamma)$ in (A.6) gives the same expression (A.5) as derived from the effective medium theory. In the effective medium theory $\gamma$ was introduced phenomenologically as the ratio between the axis of an elliptic inclusion, but the present theory shows how $\gamma$ can be obtained from the matrix $D$ involving only the surface roughness power spectrum. Note that replacing $\gamma$ with $1 / \gamma$ in (A.5) gives the result obtained using (A.6) with the other eigenvalue $\gamma /(1+\gamma)$ of the $D$-matrix. 


\section{Appendix B. Critical junction theory for anisotropic surfaces}

In earlier publications one of the present authors has studied fluid flow at interfaces using the so-called critical junction theory $[18,43]$. This theory is highly accurate at high enough contact pressures. Thus for surfaces with isotropic roughness, when the interfacial separation becomes so small that the fluid flow factor becomes of order $\sim 0.01$, which is typically the case in applications to seals, then the effective medium theory and the critical junction theory gives nearly the same result. However, the critical junction theory is much simpler to implement and require much shorter computational time even for high squeezing pressures where the contact area nearly percolate. For this latter case the effective medium theory easily becomes unstable. Here we will show how the critical junction theory can be generalized to anisotropic surfaces. Let us first briefly describe this theory in the context of leak rate of seals.

Consider the fluid leakage through a rubber seal, from a high fluid pressure $P_{\mathrm{a}}$ region, to a low fluid pressure $P_{\mathrm{b}}$ region. Assume for simplicity that the nominal contact region between the rubber and the hard countersurface is rectangular with area $L \times L$. Now, let us study the contact between the two solids as we change the magnification $\zeta$. We define $\zeta=L / \lambda$, where $\lambda$ is the resolution. We study how the apparent contact area, $A(\zeta)$, between the two solids depends on the magnification $\zeta$. At the lowest magnification we cannot observe any surface roughness, and the contact between the solids appears to be complete i.e., $A(1)=A_{0}$. As we increase the magnification we will observe some interfacial roughness, and the (apparent) contact area will decrease. At high enough magnification, say $\zeta=\zeta_{\mathrm{c}}$, a percolating path of non-contact area will be observed for the first time. The most narrow constriction along the percolation path, which we denote as the critical constriction, will have the lateral size $\lambda_{\mathrm{c}}=L / \zeta_{\mathrm{c}}$ and the surface separation at this point is denoted by $u_{\mathrm{c}}=u_{1}\left(\zeta_{\mathrm{c}}\right)$, and is given by the Persson contact mechanics theory. As we continue to increase the magnification we will find more percolating channels between the surfaces, but these will have more narrow constrictions than the first channel which appears at $\zeta=\zeta_{\mathrm{c}}$, and as a first approximation we will neglect the contribution to the leak rate from these channels.

In the critical junction theory the leak rate is obtained by assuming that all the leakage occurs through the critical percolation channel, and that the whole pressure drop $\Delta P=P_{\mathrm{a}}-P_{\mathrm{b}}$ occurs over the critical constriction (of length (in the fluid flow direction) $\lambda_{x}$ and width $\lambda_{y}$, with $\lambda_{x}=\lambda_{y}=\lambda_{\mathrm{c}} \approx L / \zeta_{\mathrm{c}}$ and height $\left.u_{\mathrm{c}}=u_{1}\left(\zeta_{\mathrm{c}}\right)\right)$. Thus for an incompressible Newtonian fluid, the volume-flow per unit time through the critical constriction will be (Poiseuille flow)

$$
\dot{Q} \approx \frac{u_{\mathrm{c}}^{3}}{12 \eta} \Delta P
$$

(a)

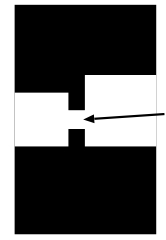

(b)

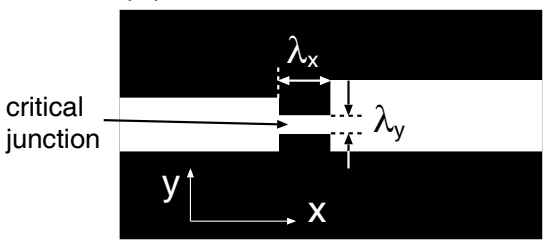

Fig. 25. The critical junction for (a) isotropic roughness and (b) anisotropic roughness (schematic).

where $\eta$ is the fluid viscosity. In deriving this equation we have assumed laminar flow and that $u_{\mathrm{c}} \ll \lambda_{c}$, which is always satisfied in practice.

To complete the theory we must calculate the separation $u_{\mathrm{c}}$ of the surfaces at the critical constriction. We first determine the critical magnification $\zeta_{c}$ by assuming that the apparent relative contact area at this point (where the non-contact area percolate) is given by the Bruggeman effective medium theory: $A\left(\zeta_{\mathrm{c}}\right) / A_{0}=0.5$. Knowing the critical magnification $\zeta_{\mathrm{c}}$, the separation $u_{c}=u_{1}\left(\zeta_{\mathrm{c}}\right)$ at the critical junction can be obtained using the Persson contact mechanics theory.

The leak rate can also be expressed in terms of the flow factor $\phi_{\mathrm{p}}$. First note that the ensemble averaged current in the $x$-direction $\bar{J}_{x}=-\left(\bar{u}^{3} \phi_{\mathrm{p}} / 12 \eta\right) \mathrm{d} \bar{p} / \mathrm{d} x$ or since $\mathrm{d} \bar{p} / \mathrm{d} x=$ $-\Delta P / L$ we get the leak rate

$$
\dot{Q}=J_{x} L=\frac{\bar{u}^{3}}{12 \eta} \phi_{\mathrm{p}} \Delta P .
$$

Comparing (B.1) and (B.2) gives

$$
\phi_{\mathrm{p}}=\left(\frac{u_{1}\left(\zeta_{\mathrm{c}}\right)}{\bar{u}}\right)^{3}
$$

For an anisotropic system $\phi_{\mathrm{p}}$ is a tensor but in an appropriately chosen coordinate system it is diagonal. We calculate can $\phi_{\mathrm{p}}$ also for anisotropic surfaces using the critical junction theory as follows. First, we assume that the non-contact area percolate at the magnification where the contact area $A / A_{0}=\gamma /(1+\gamma)$ as predicted by the Bruggeman effective medium theory. Secondly, we assume that the critical junction is rectangular in the $x y$-plane (see fig. 25) with the length (in the flow direction) width ratio $\lambda_{x} / \lambda_{y}=\gamma$. In this case the fluid flow rate through the critical junction will be

$$
\dot{Q} \approx \frac{u_{c}^{3}}{12 \eta} \lambda_{y} \frac{\Delta P}{\lambda_{x}}=\frac{u_{c}^{3}}{12 \eta} \frac{\Delta P}{\gamma},
$$

Comparing this with (B.2) gives

$$
\phi_{\mathrm{p}}=\frac{1}{\gamma}\left(\frac{u_{1}\left(\zeta_{\mathrm{c}}\right)}{\bar{u}}\right)^{3} .
$$

In fig. 26 we compare the prediction of the critical junction theory (dashed curves) with the effective medium theory prediction (solid curves). We show results for a 


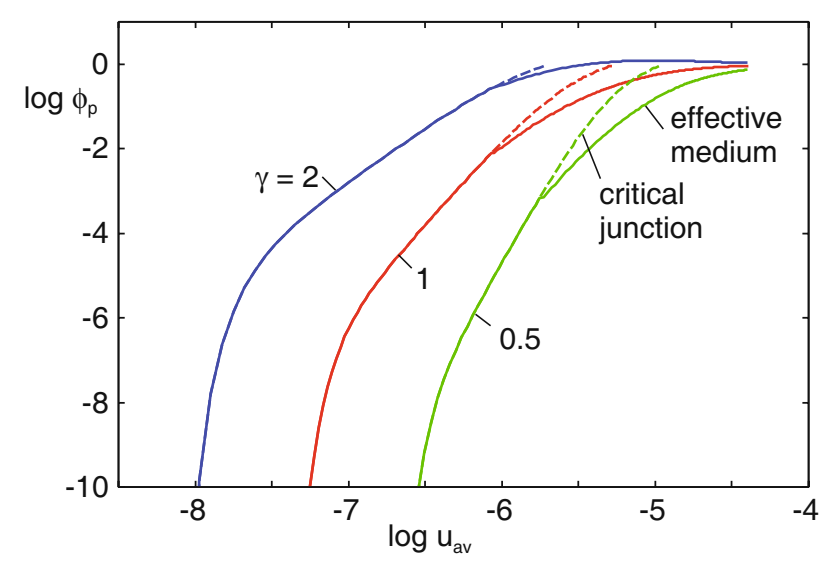

Fig. 26. The logarithm (with 10 as basis) of the pressure flow factor $\phi_{\mathrm{p}}$ as a function of the logarithm of the average interfacial separation $\bar{u}$, for anisotropic surfaces with the Peklenik numbers $\gamma=0.5,1$ and 2. The solid lines are the prediction of the effective medium theory and the dashed line the critical junction theory prediction (see text for details). In all cases the angular average power spectrum shown in fig. 4 with $H=0.9$ and the root-mean-square roughness $h_{\mathrm{rms}}=10 \mu \mathrm{m}$.

surface with isotropic roughness $(\gamma=1)$ and for two surfaces with anisotropic roughness with the Peklenik numbers $\gamma=0.5$ and $\gamma=2$. Remarkably, for small separation (or large contact pressures) the two theories give nearly the same result in all cases. The critical junction theory can even be applied to the case where the Peklenik function depends on the magnification (see sect. 2). In this case one should use in the theory the Peklenik number $\gamma=\gamma\left(\zeta_{\mathrm{c}}\right)$ for the magnification $\zeta_{\mathrm{c}}$ where the non-contact area first percolate. Thus, $\zeta_{\mathrm{c}}$ is determined by the condition $A(\zeta) / A_{0}=\gamma(\zeta) /(1+\gamma(\zeta))$. The orientation of the coordinate system in which $\phi_{\mathrm{p}}$ is diagonal is then determined by the Peklenik angle $\psi\left(\zeta_{\mathrm{c}}\right)$.

\section{References}

1. N. Patir, H.S. Cheng, J. Tribol., Trans. ASME 100, 12 (1978).

2. N. Patir, H.S. Cheng, J. Tribol., Trans. ASME 101, 220 (1979).

3. J.A. Greenwood, J.B.P. Williamson, Proc. R. Soc. London, Ser. A 295, 300 (1966).

4. A.W. Bush, R.D. Gibson, T.R. Thomas, Wear 35, 87 (1975).

5. B.N.J. Persson, J. Phys.: Condens. Matter 20, 312001 (2008).

6. C. Campañá, M.H. Müser, M.O. Robbins, J. Phys.: Condens. Matter 20, 354013 (2008).

7. B.N.J. Persson, Phys. Rev. Lett. 99, 125502 (2007).

8. C. Yang, B.N.J. Persson, J. Phys.: Condens. Matter 20, 215214 (2008).

9. B. Lorenz, B.N.J. Persson, J. Phys.: Condens. Matter 201, 015003 (2009).
10. F. Sahlin, A. Almqvist, R. Larsson, S. Glavatskih, Tribol. Int. 40, 1025 (2007).

11. J.H. Tripp, ASME J. Lubrication Technol. 105, 485 (1983).

12. M. Scaraggi, B.N.J. Persson, G. Carbone, D. Dini, Soft Matter 7, 10395 (2011).

13. B.N.J. Persson, J. Phys.: Condens. Matter 22, 265004 (2010).

14. B.N.J. Persson, O. Albohr, U. Tartaglino, A.I. Volokitin, E. Tosatti, J. Phys.: Condens. Matter 17, R1 (2005).

15. D. Bruggeman, Ann. Phys. (Leipzig) 24, 636 (1935).

16. S. Kirkpatrick, Rev. Mod. Phys. 45, 574 (1973).

17. M. Sahimi, Heterogeneous Materials I (Springer, New York, 2003).

18. B. Lorenz, B.N.J. Persson, Eur. Phys. J. E 31, 159 (2010).

19. B.N.J. Persson, J. Chem. Phys. 115, 3840 (2001).

20. B.N.J. Persson, Surf. Sci. Rep. 61, 201 (2006).

21. A. Almqvist, C. Campañá, N. Prodanov, B.N.J. Persson, J. Mech. Phys. Solids 59, 2355 (2011).

22. B. Lorenz, B.N.J Persson, J. Phys.: Condens. Matter 23, 355005 (2011).

23. B. Lorenz, B.N.J. Persson, Eur. Phys. J. E 32, 281 (2010).

24. W. Humphrey, A. Dalke, K. Schulten, J. Molec. Graph. 14, 33 (1996).

25. C. Yang, U. Tartaglino, B.N.J. Persson, Eur. Phys. J. E 19, 47 (2006).

26. M. Griebel, S. Knapek, G. Zumbusch, Numerical Simulation in Molecular Dynamics (Springer, Berlin, Heidelberg, 2007).

27. D.C. Rapaport, The Art of Molecular Dynamics Simulation, second edition (Cambridge University Press, Cambridge, 2004).

28. Y. Mo, K.T. Turner, I. Szlufarska, Nature 457, 1116 (2009).

29. J. Schmelzer Jr., S.A. Brown, A. Wurl, M. Hyslop, R.J. Blaikie, Phys. Rev. Lett. 88, 226802 (2002).

30. A.D. Pogrebnjak, A.P. Shpak, N.A. Azarenkov, V.M. Beresnev, Phys. Usp. 52, 29 (2009).

31. A. Kapitulnik, A. Aharony, G. Deutscher, D. Stauffer, J. Phys. A: Math. Gen. 16, L269 (1983).

32. W.J. Boudville, T.C. McGill, Phys. Rev. B 39, 369 (1989).

33. C. Vanneste, A. Gilabert, D. Sornette, Phys. Lett. A 155, 174 (1991).

34. A.I. Olemskoi, V.F. Klepikov, Phys. Rep. 338, 571 (2000).

35. B.A. Krick, J.R. Vail, B.N.J. Persson, W.G. Sawyer, Tribol. Lett. 45, 185 (2011).

36. B.N.J. Persson, Eur. Phys. J. E 8, 385 (2002).

37. N. Rathore, P. Pranay, B. Eu, W. Ji, E. Wallis, PDA J. Pharm. Sci. Tech. 65, 468 (2011).

38. S. Yamada, Tribol. Lett. 13, 167 (2002).

39. L. Bureau, Phys. Rev. Lett. 104, 218302 (2010).

40. B. Lorenz, B.N.J. Persson, S. Dieluweit, T. Tada, Eur. Phys. J. E 34, 129 (2011).

41. I. Sivebaek, V. Samoilov, B.N.J. Persson, Phys. Rev. Lett. 108, 036102 (2012).

42. B.N.J. Persson, F. Mugele, J. Phys.: Condens. Matter 16, R295 (2004).

43. B.N.J. Persson, C. Yang, J. Phys.: Condens. Matter 20, 315011 (2008). 